\title{
Ceramidase Regulates Synaptic Vesicle Exocytosis and Trafficking
}

\author{
Jeffrey Rohrbough, ${ }^{1}$ Emma Rushton, ${ }^{1}$ Laura Palanker, ${ }^{2}$ Elvin Woodruff, ${ }^{1}$ Heinrich J. G. Matthies, ${ }^{1}$ Usha Acharya, ${ }^{3}$ \\ Jairaj K. Acharya, ${ }^{3}$ and Kendal Broadie ${ }^{1}$ \\ ${ }^{1}$ Department of Biological Sciences, Vanderbilt Kennedy Center, Vanderbilt Brain Institute, Vanderbilt University, Nashville, Tennessee 37235-1634, \\ ${ }^{2}$ Department of Biology, University of Utah, Salt Lake City, Utah 84112, and ${ }^{3}$ Regulation of Cell Growth Laboratory, National Cancer Institute-Frederick, \\ Frederick, Maryland 21702
}

\begin{abstract}
A screen for Drosophila synaptic dysfunction mutants identified slug-a-bed(slab). The slab gene encodes ceramidase, a central enzyme in sphingolipid metabolism and regulation. Sphingolipids are major constituents of lipid rafts, membrane domains with roles in vesicle trafficking, and signaling pathways. Null slab mutants arrest as fully developed embryos with severely reduced movement. The SLAB protein is widely expressed in different tissues but enriched in neurons at all stages of development. Targeted neuronal expression of $s$ lab rescues mutant lethality, demonstrating the essential neuronal function of the protein. $\mathrm{C}_{5}$-ceramide applied to living preparations is rapidly accumulated at neuromuscular junction (NMJ) synapses dependent on the SLAB expression level, indicating that synaptic sphingolipid trafficking and distribution is regulated by SLAB function. Evoked synaptic currents at slab mutant NMJs are reduced by $50-70 \%$, whereas postsynaptic glutamate-gated currents are normal, demonstrating a specific presynaptic impairment. Hypertonic saline-evoked synaptic vesicle fusion is similarly impaired by $50-70 \%$, demonstrating a loss of readily releasable vesicles. In addition, FM1-43 dye uptake is reduced in slab mutant presynaptic terminals, indicating a smaller cycling vesicle pool. Ultrastructural analyses of mutants reveal a normal vesicle distribution clustered and docked at active zones, but fewer vesicles in reserve regions, and a twofold to threefold increased incidence of vesicles linked together and tethered at the plasma membrane. These results indicate that SLAB ceramidase function controls presynaptic terminal sphingolipid composition to regulate vesicle fusion and trafficking, and thus the strength and reliability of synaptic transmission.
\end{abstract}

Key words: Drosophila; synaptic transmission; synapse; presynaptic; ceramidase; ceramide; sphingolipids; lipid regulation

\section{Introduction}

Membrane lipid composition and lipid-based signaling are critical to neuronal function. Synaptic membranes are heterogeneously distributed into specialized domains, ensuring specific lipid and protein interactions required for regulated neurotransmission. Whereas neuronal plasma membrane (PM) and vesicle lipids consist predominantly of phospholipids and cholesterol, sphingolipids, including sphingomyelin, ceramide, and related glycosphingolipids, serve essential structural, modulatory, and signaling roles in surface and internal membranes (Merrill et al., 1997; van Meer and Holthuis, 2000; Hoekstra et al., 2003). Sphingolipids have compact head groups and relatively saturated acyl tails, promoting lipid order and packing, and self-aggregate with cholesterol into lipid raft domains (Brown and London, 2000; Lai, 2003; van Blitterswijk et al., 2003). Lipid rafts are platforms

Received March 27, 2004; revised July 11, 2004; accepted July 13, 2004.

This work was supported by National Institutes of Health Grant GM54544 to K.B. We gratefully acknowledge the Bloomington Drosophila Stock Center and the lowa Hybridoma Bank for essential genetic and antibody reagents, respectively. We are particularly grateful to T. Fergestad and B. Aravamudan for technical advice and feedback and to C. Rodesch and Y. Zhang for technical assistance.

Correspondence should be addressed to Dr. Kendal S. Broadie, Department of Biological Sciences, Vanderbilt University, VU Station B, Box 351634, Nashville, TN 37235-1634. E-mail: kendal.broadie@vanderbilt.edu. D0I:10.1523/JNEUROSCI.1146-04.2004

Copyright $\odot 2004$ Society for Neuroscience $\quad 0270-6474 / 04 / 247789-15 \$ 15.00 / 0$ for protein targeting and sorting, vesicle endocytosis, fusion and trafficking, actin cytoskeleton regulation, and diverse cellular signaling pathways (Brown and London, 2000; Maekawa et al., 2003; Helms and Zurzolo, 2004).

Ceramide is particularly important in membrane domain formation and vesicle trafficking. Ceramide is synthesized in the endoplasmic reticulum (ER) and transported to the PM and generated in the PM by sphingomyelin hydrolysis (van Blitterswijk et al., 2003; Riezman and van Meer, 2004). In the outer PM leaftet, ceramide aggregates into lateral domains in association with sterols and other sphingolipids, aiding raft stabilization (Venkataraman and Futerman, 2000; van Blitterswijk et al., 2003). Ceramidase, a central enzyme in sphingolipid metabolism and signaling, cleaves ceramide to produce the motile second messenger sphingosine. Neutral/alkaline ceramidases (Cdases) share the greatest conservation among disparate organisms. Mammalian neutral Cdases are secreted but localized to the PM by $O$-glycosylation of a serine-threonine-rich mucin domain absent in invertebrate homologs (Tani et al., 2003). Perturbing Cdase activity disrupts cholesterol and sphingolipid distribution (Pagano et al., 2000a) and raft protein sorting (McMaster, 2001; Watanabe et al., 2002) and modulates endocytic pathways in the Drosophila retina (Acharya et al., 2003, 2004).

Lipid rafts have increasingly recognized roles in synaptic do- 
main organization and signaling processes (Martin, 2000; Paratcha and Ibanez, 2002; Tsui-Pierchala et al., 2002). Lipid topology is relevant for synaptic morphological specialization and the extreme membrane structural changes accompanying synaptic vesicle (SV) endocytosis and fusion (van Blitterswijk et al., 2003). Rafts localize and functionally modulate certain neuronal ion channels and neurotransmitter receptors (Bruses et al., 2001; Suzuki et al., 2001; Tsui-Pierchala et al., 2002; Eroglu et al., 2003; Hering et al., 2003; Taverna et al., 2004) and regulate postsynaptic morphology (Hering et al., 2003). In particular, raft lipid and protein interactions potentially regulate neurotransmitter release. Raft and raft-like domains localize essential components of the vesicular exocytic machinery, including syntaxin, SNAP-25 (soluble $N$-ethylmaleimide-sensitive factor attachment protein), and synaptobrevin (Lafont et al., 1999; Chamberlain et al., 2001; Lang et al., 2001; Chamberlain and Gould, 2002). SV recycling may also entail recruitment of endocytic machinery to preassembled domains concentrated in sphingolipids, cholesterol, and SV proteins (Martin, 2000; Mitter et al., 2003). Additionally, multiple proteins regulating SV pool organization and mobilization, including F-actin, synapsin, and synaptophysin, bind or interact directly with SV lipids (Benfenati et al., 1992; Greengard et al., 1993, 1994; Ceccaldi et al., 1995; Thiele et al., 2000; Bloom et al., 2003; Sankaranarayanan et al., 2003).

The Drosophila neuromuscular junction (NMJ) is a well studied model for investigating SV trafficking and transmitter release mechanisms (Richmond and Broadie, 2002; Kidokoro, 2003). The homology between Drosophila and vertebrate raft composition and function (Rietveld et al., 1999) predicts that genetic and functional analysis in this system will provide insight into the roles of sphingolipids and rafts in synaptic regulation. We identified slug-a-bed (slab) in a forward screen for novel synaptic dysfunction mutants. The slab gene encodes a long-chain Cdase (Yoshimura et al., 2002) essential in the nervous system. Null slab mutant embryos characteristically arrest partially hatched from the egg case, thus appearing disinclined to get moving (hence "slug-a-bed"; see Romeo and Juliet: scene V). Mutant NMJs have impaired presynaptic transmitter release and a reduced cycling SV pool. Ultrastructurally, slab terminals have normally clustered and docked SVs at active zones (AZs), but fewer SVs overall, and increased tethering of vesicles together and to the PM, indicating specific defects in SV fusion and trafficking. These results reveal an essential role for SLAB Cdase in regulating sphingolipiddependent SV fusion and trafficking processes underlying neurotransmission.

\section{Materials and Methods}

Genetics and Drosophila stocks. The slab ${ }^{1}$ mutation was generated in an ethyl methanesulfonate (EMS) screen of an isogenized rucuca $(r u, h, t h$, $s t, c u, s r, e, c a$ ) third chromosome (Featherstone et al., 2000). For mapping and functional analyses, deficiencies in the 93-100 region were obtained from the Third Chromosome Deficiency kit (Bloomington Drosophila Stock Center, Bloomington, IN). Df(3R)20 was a gift from ZhiChun Lai (Pennsylvania State University, University Park, PA). Other slab alleles were generated by local hop P-element mutagenesis (Grigliatti, 1998), using $\mathrm{P}\{\mathrm{lacW}\} 1(3){ }^{38 B} 9^{\text {j8B9 }}$ (Bloomington Drosophila Stock Center). The j8B9 flies were crossed to $\Delta 2-3 \mathrm{CyO} / \mathrm{Bc}$, and male progeny containing both transposon and transposase were crossed singly to $w$; $L y / T M 6 T b$ virgins (66 crosses). Male progeny lacking the $\Delta 2-3 C y O$ chromosome were mated singly to $w$; slab $/ T M 6 S b, T b$ virgins (309 crosses). Seven new independent P-element insertion lines were identified based on failure to complement $s l a b^{1}$. An adjacent sequence was cloned by plasmid rescue (O'Kane, 1998) and identified by a BLAST (Basic Local Alignment Search Tool) search of the Drosophila genome database (Adams et al., 2000). The $s l a b^{2}$ mutation contains an 855 bp deletion spanning exons 4 and 5 of slab (CG1471). The slab ${ }^{3}$ mutation deletes the slab and CG2224 genes, and portions of adjacent genes aralar and PH4alphaEFB (Adams et al., 2000). Other slab alleles included larger deletions, in each case with the $\mathrm{P}$ element $5^{\prime}$ (downstream) segment retaining its original position in 1(3)j8B9 and the $3^{\prime}$ (upstream) segment adjacent to upstream genomic DNA.

To map deletions, homozygous slab mutant embryos were selected by the absence of the green fluorescent protein (GFP) balancer TM3, P\{GAL4-Kr.C\}DC2, P\{UAS-GFP.S65T\}DC10, Sb. Single-embryo PCR was performed on four homozygous mutant embryos and two balanced embryos for each allele. The primer pair CGGCAATGAGTGTGATCTAC and GTTGCGCATTAAGTGATGACC, which generate an 824 base pair fragment from the coding region of CG1471, was used for screening. These primers produced no band for any single homozygous embryos. Control primers specific for a region of genomic scaffold AE003678 produced a band in each case. To identify the $s l a b^{1}$ mutation, homozygous $s l a b^{1}$ embryos were selected by the absence of the GFP balancer, and RNA was prepared using TriZol (Invitrogen, San Diego, CA). The cDNA was prepared using the Ominiscript kit (Qiagen, Chatsworth, CA), amplified by PCR using Platinum Pfx (Invitrogen), and the resulting DNA, as well as control cDNA from parental rucuca flies, was sequenced. Homozygous and hemizygous $s l a b^{1}, s l a b^{2}$, and $s l a b^{3}$ alleles were used for characterization of mutant morphological, functional, and ultrastructural phenotypes and, in all cases, selected at late embryonic stages by the absence of GFP. Controls included wild-type (Oregon R) and $s l a b^{1}$ heterozygotes balanced over TM3 Sb Kr-GFP (indicated in the text as slab ${ }^{1} / T M 3$ ).

Bioinformatics. Entrez-PubMed searches were performed at the National Center for Biotechnology Information website (http://www.ncbi.nlm.nih.gov/pubmed). BLASTP searches were performed using search queries for Drosophila CG1471, human acid Cdase (Farber's disease, AAC50907), and the C. elegans conjectural proteins 04586 and Q09551. Ceramidase sequences were analyzed using CLUSTALW at the Center for Molecular and Biomolecular Informatics website.

Generation and transformation of transgenic animals. A construct containing the full-length genomic slab (CG1471) sequence was generated by PCR of Oregon R genomic DNA with the Expand Long Template system (Roche, Indianapolis, IN). The construct includes 3553 bases upstream of the translation start and the complete genomic coding region. The fragment was cut with EagI and XhoI and ligated into pUAST (http://flybase.bio.indiana.edu). Constructs of UAS-slab and UAS-slab fused to the coding sequence of enhanced GFP (eGFP) were generated by PCR of the genomic coding region. For the UAS-slab-eGFP construct, the stop codon was omitted from the fragment. These fragments were similarly cut and ligated into pUAST-GFP. All three constructs were transformed into DH10B electrocompetent cells (Invitrogen) and isolated in concentrated and purified form for microinjection with a transposase $(\Delta 2-3)$. Embryos $\left(w^{1118}\right)$ were injected for transformation using standard techniques, and progeny were screened for $w^{+}$expression to identify stable insertions of the transgene.

The third chromosomes carrying the full-length slab-eGFP transgene and either the $s l a b^{1}$ or $s l a b^{2}$ mutations were made by recombination. The presence of the slab mutation was confirmed by PCR and sequencing. UAS-slab and UAS-slab-eGFP were driven using P\{GAL4-da.G32\}UH1 (UH1 gal4) (Wodarz et al., 1995) and elav gal4 (Luo et al., 1994). Genotypes of rescued flies were elav gal4; UAS-slab, slab ${ }^{1} /$ sla $^{2}$; and UH1 gal4, slab ${ }^{2} /$ UAS-slab, slab ${ }^{1}$.

Apoptosis assays. Wild-type and $s l a b^{1} / s l a b^{3}$ mutant embryos were selected at early stage $16\left[\sim 13 \mathrm{hr}\right.$ after egg laying (AEL) at $\left.25^{\circ} \mathrm{C}\right]$. Embryos were treated for $5 \mathrm{~min}$ at room temperature with $5 \mu \mathrm{g} / \mathrm{ml}$ acridine orange (Molecular Probes, Eugene, OR) in PBS, 1:1 with heptane, mounted in halocarbon oil (Abrams et al., 1993), and oriented on their sides. Z-series confocal images were made with a LSM 510 microscope (Zeiss, Oberkochen, Germany), using a $488 \mathrm{~nm}$ argon excitation laser. Each series sectioned approximately halfway through the embryo, using identical slice thickness and number for each embryo. Bright spots indicating apoptotic cells were counted and summed in 17 slices for each embryo.

Immunohistology. Immunocytochemistry of mature control and mutant embryos was performed as described previously (Featherstone et al., 
2002). Central neurons, peripheral nerves, and presynaptic NMJ terminals were visualized with Texas Red- or FITC-conjugated antihorseradish peroxidase (HRP; 1:200; Molecular Probes). Presynaptic vesicle staining was examined with anti-synaptotagmin I (1:200) and Alexa-conjugated secondary antibody (1:500). Glutamate receptor clusters were visualized with anti-GluRIIA antibody (1:20; Iowa Hybridoma Bank, University of Iowa, Iowa City, IA) and Alexa 488-conjugated secondary antibody (1:500; Molecular Probes). Anti-SLAB staining was done in mature embryos and third instar larvae using rabbit polyclonal antibodies against the N-terminal (Ab11.3) or C-terminal (Ab10.3) half of the protein or mouse monoclonal antibodies, at dilutions of 1:200-1: 500 , and visualized with the appropriate Alexa-conjugated secondary antibody. Confocal images of the CNS, neuromusculature, and additional tissues were acquired and processed with Adobe Photoshop software. NMJ presynaptic terminal areas were measured at muscles 12 and 13 in anti-HRP-stained wild-type and mutant embryonic preparations. Terminal regions were outlined, and area measurements were made using NIH Image (total pixels); areas for one to two NMJs in five animals were averaged for each genotype.

Western analysis. Sixty embryos or 10 fly heads were collected from the appropriately identified genotypes and homogenized in sample buffer containing $2 \times$ complete protease inhibitors. Extract from 60 embryos or two fly heads were electrophoresed using 5-20\% SDS-PAGE gradient gels and transferred to polyvinylidene difluoride. Ceramidase antibody staining (1:1000 dilution) was performed in PBS-Tween-4\% powdered milk. Blots were developed by using alkaline phosphatase-conjugated secondary antibodies with 5-bromo-4-chloro-3-indolyl phosphate and nitroblue tetrazolium as substrates.

Fluorescent ceramide staining and analyses. Staged embryos (22-24 hr AEL) or newly hatched larvae, or mature third instar larvae, were dissected in low $\left[\mathrm{Ca}^{2+}\right]$ recording saline (see below) and transferred to Schneiders Drosophila medium (Invitrogen, Gaithersburg, MD). Preparations were incubated for $30-40 \mathrm{~min}$ at $18^{\circ} \mathrm{C}$ with $10 \mu \mathrm{M}$ BODIPY $\mathrm{Fl}$ $\mathrm{C}_{5}$-ceramide $\left(\mathrm{C}_{5}\right.$-Cer) complexed to BSA (Molecular Probes) in Schneiders medium, rinsed several times with fresh medium, and then washed for 1-2 hr, with several medium changes, in the dark at room temperature. Transgenic Fl-slab animals were processed in parallel with slab ${ }^{3}$ TM3 heterozygotes and/or wild-type larvae in the same chamber in each experiment. Confocal images of BODIPY $\mathrm{C}_{5}$-Cer fluorescence were collected using a $40 \times$ or $63 \times$ water immersion lens, using identical confocal settings for each set of preparations to allow direct comparison of fluorescence among genotypes. Emitted fluorescence was acquired with a 560 $\mathrm{nm}$ long-pass filter. Emission spectra of BODIPY-labeled sphingolipids is shifted from green to red wavelengths ( $\sim 617 \mathrm{~nm}$ emission peak) with increasing membrane concentration, allowing regions of accumulated BODIPY-lipid to be distinguished (Pagano et al., 1991, 2000a,b). To confirm synaptic localization of fluorescent sphigolipid, preparations were fixed (4\% paraformaldehyde for $30 \mathrm{~min}$ at room temperature) after live imaging, stained with rabbit anti-Discs large (DLG; 1:500) and Alexa 488-conjugated secondary antibodies (Beumer et al., 2002), and mounted between two coverslips in Vectashield (Vector Laboratories, Burlingame, CA). The same NMJs were reimaged ( $40 \times$ oil objective) to compare synaptic $\mathrm{C}_{5}$-Cer and DLG localization. The synaptic $\mathrm{C}_{5}$-Cer fluorescence level was quantified using NIH Image software. Contiguous regions of stained synaptic terminals (NMJ 4) were outlined, and mean brightness (1-256 grayscale) was measured for the outlined region. Several NMJs were averaged for each preparation, and the average value in each experiment was normalized to that of Fl-slab.

Electrophysiology. Physiological recordings were made at the NMJ of staged embryos and newly hatched larvae, as described previously (Broadie and Bate, 1993). Unhatched embryos were dechorionated in bleach and removed manually from the vitelline membrane case. Animals were transferred to recording saline, secured at the head and tail to sylgard-coated coverslips using surgical histoacryl glue, dissected open dorsally, and glued flat. Preparations were exposed to collagenase (1.0 $\mathrm{mg} / \mathrm{ml}$, type IV; Sigma, St. Louis, MO) in $0.2 \mathrm{mM} \mathrm{Ca}^{2+}$-containing saline for 1-2 min and washed with fresh recording saline. The bath recording saline composition contained (in $\mathrm{mm}$ ): $135 \mathrm{NaCl}, 5 \mathrm{KCl}, 1.8 \mathrm{CaCl} 2,4$ $\mathrm{MgCl}$, 5 TES, 36 sucrose, and $2 \mathrm{NaOH}, \mathrm{pH}$ 7.2.
Standard whole-cell patch-clamp recordings at a holding potential of $-60 \mathrm{mV}$ were made from muscle 6 in anterior abdominal segments (A2-A4). Excitatory junctional currents (EJCs) were evoked by peripheral nerve stimulation using a glass suction electrode, or by central stimulation using a $3 \mathrm{M} \mathrm{KCl}$-filled sharp electrode placed in the center of the ventral nerve cord (Yoshihara et al., 2000). Both stimulation techniques produced similar EJC amplitudes, but nerve stimulation was used in the majority of recordings because central stimulation tended to promote segmental muscle contraction. Glutamate (100 mM in dH20, pH 9) was iontophoretically applied from sharp microelectrodes positioned in the middle of the muscle 6/7 NMJ (Broadie and Bate, 1993). Brief glutamate applications of 2-10 msec elicited currents with highly reproducible peak amplitudes and $<500 \mathrm{msec}$ duration. Hyperosmotic (HO) saline consisted of normal recording saline with $850 \mathrm{~mm}$ of added sucrose (Aravamudan et al., 1999; Fergestad and Broadie, 2001). HO saline was pressure ejected $(2 \mathrm{sec}$ ) from an unpolished patch pipette onto the muscle $6 / 7$ NMJ using low (1-2 psi) pressure to reduce movement artifacts and nonsynaptic muscle conductance. The ejected stream was observed visually to encompass the entire NMJ. The number of discrete current peaks in the response and the synaptic charge (measured as area under the current trace relative to baseline, in units of pAS) were quantified for 4 sec beginning with the start of the application.

Postsynaptic currents were filtered (1-2 kHz) and acquired to disk (5 $\mathrm{kHz}$ sampling) using hardware and computer interface from Axon Instruments (Foster City, CA) and analyzed with PClamp version 6 or version 8 software. Representative data traces were exported and prepared for display using Igor or standard spreadsheet and graphics software.

FM1-43 dye imaging. Hatching-stage (22-24 hr AEL) control and mutant embryos were dissected in $\mathrm{Ca}^{2+}$-free recording saline. One control and one mutant embryo were always prepared on the same coverslip to ensure identical processing and imaging conditions (Fergestad and Broadie, 2001). Confocal settings underwent little or no variation between experiments. Embryos were exposed to $10 \mu \mathrm{M}$ FM1-43 (Molecular Probes) in $90 \mathrm{~mm} \mathrm{~K}^{+}$saline containing $1.8 \mathrm{~mm} \mathrm{Ca}^{2+}$ (Fergestad and Broadie, 2001) for $2.5 \mathrm{~min}$ to load synaptic terminals, then washed in $\mathrm{Ca}^{2+}$-free recording saline $\left(2 \mathrm{mM} \mathrm{K}^{+}\right)$for a minimum of $5 \mathrm{~min}$ to remove background fluorescence. Confocal fluorescence images were acquired with a $63 \times$ water lens. In each animal, one to two loaded muscle 12 and 13 NMJs were imaged (segments A2-A4) and then re-imaged after a second high $\mathrm{K}^{+}$treatment to destain terminals. Successive images for each NMJ were exported into Adobe Photoshop and aligned precisely as layers and quantified as a multi-image stack in NIH Image. The density slice function, adjusted to twofold to threefold over background, was used to outline the loaded terminal boutons $(>1 \mu \mathrm{m})$, and the identical region was superimposed onto the aligned destained terminals. Mean synaptic loading intensity was measured for each loaded and destained NMJ 12/13 pair. Background fluorescence from a nonsynaptic region of muscle surface was subtracted from loaded and destained synaptic levels, and values from multiple NMJs were averaged for each animal. Normalized, background-subtracted pixel intensities are reported.

Electron microscopy and ultrastructural analysis. Mutant $\left(\operatorname{slab}^{1} /\right.$ slab $\left.^{3}\right)$ and control (wild-type or slab/TM3) embryos from timed egg lays were collected, fixed, sectioned, and visualized in parallel using standard transmission electron microscopy techniques, as reported previously (Broadie et al., 1995; Featherstone et al., 2001). Briefly, embryos were dechorionated, removed from the vitelline membrane, and the anterior and posterior ends were excised in 5\% glutaraldehyde in $0.05 \mathrm{~m}$ PBS. Embryos were then transferred to $2.5 \%$ glutaraldeyde in $0.05 \mathrm{M}$ PBS for 1 $\mathrm{hr}$, washed three times in PBS, transferred to $1 \% \mathrm{OsO}_{4}$ in distilled water $\left(\mathrm{dH}_{2} \mathrm{O}\right)$ for $1 \mathrm{hr}$, and washed three times in $\mathrm{dH}_{2} \mathrm{O}$. Preparations were then stained en bloc in $1 \%$ aqueous uranyl acetate for $1 \mathrm{hr}$, washed three times in $\mathrm{dH}_{2} \mathrm{O}$, dehydrated in an ethanol series $(30-100 \%)$, passed through propylene oxide, transferred to a 1:1 araldite:propylene oxide mixture, and removed and embedded in Epon 812 epoxy resin. Ultrathin serial sections $(50-60 \mathrm{~nm}$ ) were obtained on a UCT Ultracut microtome (Leica, Nussloch, Germany), transferred to formvar-coated grids, and examined on a Phillips CM12 transmission electron microscope. Synaptic boutons were serially sectioned, and profiles for each identified bouton were quantified in the section where an electron-dense AZ and T-bar 
structure were most prominent. Only sections containing a single $\mathrm{AZ}$ were quantified. SVs in the "clustered" pool were defined as those within $250 \mathrm{~nm}$ of an AZ. Docked vesicles were defined as those within 0.5 vesicle diameter $(<20 \mathrm{~nm})$ of the electron-dense PM at the AZ. Measurements and quantification were made using Image J. Docked, clustered, and total vesicles were scored for each profile, as well as vesicles linked together or tethered to the PM outside the clustered zone, by electron-dense material. Linked and tethered vesicles were also examined in one to two serial sections adjacent to the principle AZ-containing section. Mean quantified parameters were statistically compared using the Mann-Whitney test, and presentation images were processed in Adobe Photoshop.

\section{Results}

Identification and characterization of the slug-a-bed Cdase gene

An EMS mutagenesis screen of the third chromosome (comprising $\sim 40 \%$ of the Drosophila genome) was performed to identify novel molecules essential for neurotransmission. Mutant lines resulting in late embryonic or early postembryonic lethality were sequentially screened for normal gross anatomy, impaired movement, correctly patterned neuromusculature, and finally for synaptic dysfunction using whole-cell patch-clamp recordings at the embryonic NMJ (Broadie and Bate, 1993). The EMSinduced $s l a b^{1}$ mutation was isolated on an isogenized rucuca $(r u, h, t h, s t, c u, s r, e, c a)$ chromosome and mapped by recombination with wild type (Oregon $\mathrm{R}$ ) to a region distal to the $e$ locus. Genomic deficiency mapping showed that $s l a b^{1}$ fails to complement Df(3R)tll-g (99F1-2;100B5) but complements Df(3R)20 (100A1-2;100B1-2), placing the slab locus in 99F1-2;100A1-2 or 100B1-2;100B5 (Fig. 1A).

All available lethal P-element insertions in the region complement slab ${ }^{1}$. To create additional mutant alleles and to facilitate cloning the gene, $\mathrm{P}\{\mathrm{lacW}\} 1(3) \mathrm{j} 8 \mathrm{~B} 9^{\mathrm{j} 8 \mathrm{~B} 9}$ (j8B9) was mobilized in a P-element local hop mutagenesis. New insertions were complementation tested for lethality against slab ${ }^{1}$ to isolate seven new slab mutant alleles. All insertion alleles retain a $5^{\prime}$ (distal) $\mathrm{P}$ element sequence adjacent to prolyl-4-hydroxylase- $\alpha$ EFB (PH $4 \alpha$ EFB), the original insertion site of j8B9 (Fig. $1 B$ ). Six insertion alleles have a 3' (proximal) P-element sequence adjacent to genes proximal to j8B9 (Fig. $1 B$ and data not shown). PCR on single homozygous embryos with CG1471-specific primers verified that the CG1471 sequence was deleted in three of these lines. The $s l a b^{2}$ deletion allele has an intragenic deletion of 855 bases removing parts of exons 4 and 5; slab ${ }^{2}$ retains its original insertion site both $5^{\prime}$ and $3^{\prime}$ of the j8B9 P-element and has a new insertion in CG1471 (Fig. 1B). A second larger deletion allele, $s_{a b}{ }^{3}$, removes CG1471 and CG2224 and portions of the adjacent genes aralar and PH4 $\alpha$ EFB (Fig. 1B). CG1471 maps to 99F5-99F6

A Deficiency map

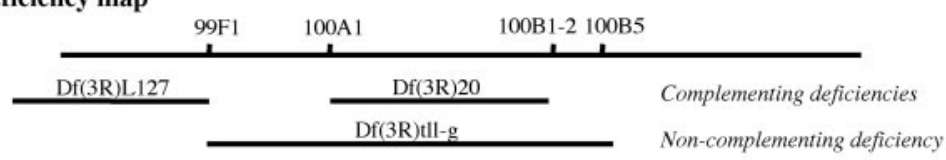

B Molecular organization of slab region
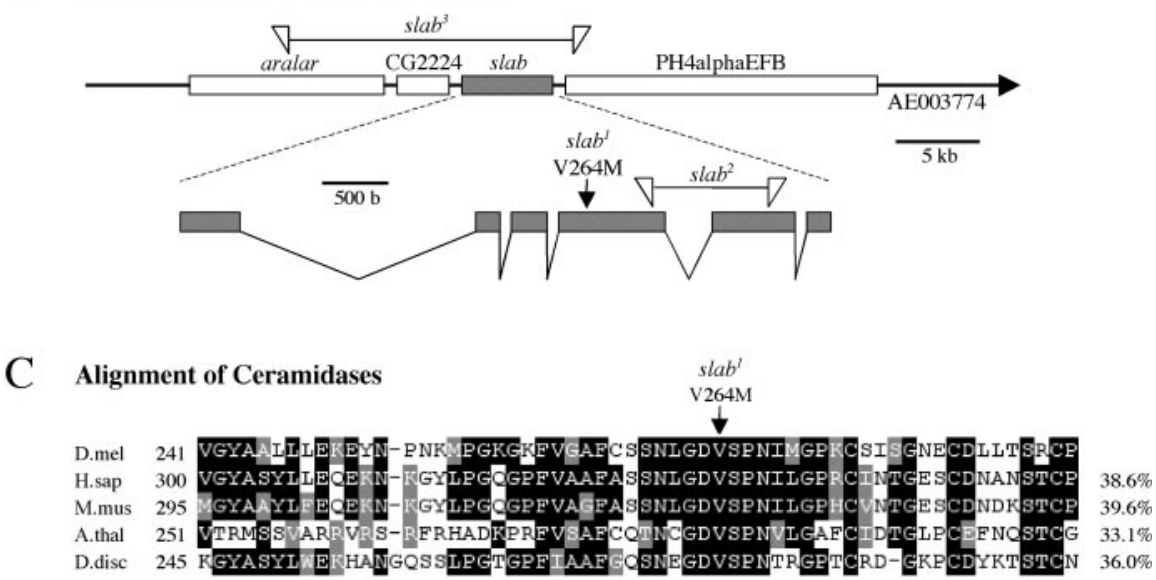

\section{Phylogeny of Ceramidases}

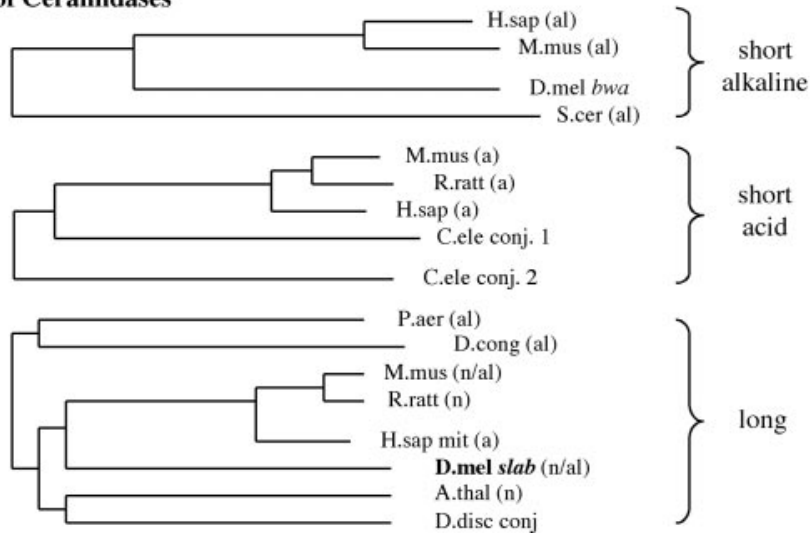

Figure 1. Genomic mapping and identification of the slug-a-bed ceramidase gene and mutants. A, Genomic map showing the right tip of chromosome 3R. Genomic regions deleted by complementing and noncomplementing deficiency (Df) lines are represented below. B, Molecular organization of the slab locus, schematizing genomic scaffold AE003774. The boxes represent known and predicted genes in the region, with slab indicated by the shaded box. The deleted sequence in the sla $b^{3}$ mutation (line and triangles) is indicated above; the right triangle is the original point of insertion of $\mathrm{j8B9}$. The expanded region below shows the intron/exon structure of slab to scale. The site of the $1 a b^{7}$ point mutation (arrow) and the region deleted in the slab mutation are indicated. C, CLUSTALW alignment of five related (dases, with overall homology to D.mel SLAB Cdase indated by the percentages at right: human mitochondrial acid (H.sap), mouse alkaline/neutral (M.mus), Arabidopsis neutral (A.thal), and Dictyostelium predicted gene "random slug CDNA25 protein" (D.disc) Cdases. The slab" point mutation occurs in a highly conserved valine (arrow). D, Phylogenetic trees (midpoint rooted) showing relationship of (dases. al, Alkaline; n, neutral; a, acid; conj, conjectural (predicted from a genome sequence annotation). In addition to Cdases listed in C, related proteins include rat neutral (R.ratt), Dermatophilus congolensis alkaline (D.cong), and Pseudomonas alkaline (P.aer) Cdases. D.mel bwa is the gene brainwashing, and H.sap acid Cdase is implicated in Farber's disease.

(The FlyBase Consortium, 2003), consistent with the deficiency mapping data. The $\operatorname{slab}^{1}$ (EMS) mutation results in a single point mutation of valine 264 to methionine in exon 4 of CG1471 (Fig. $1 B, C)$.

BLAST searches against sequenced genomes revealed CG1471 to be a recently identified Drosophila Cdase (Renault et al., 2002; Yoshimura et al., 2002), with demonstrated functional Cdase activity when expressed in Drosophila S2 cultured cells (Yoshimura et al., 2002). Comparison among different organisms reveals three major groups of Cdases; the best characterized and most highly conserved are "long" Cdases (670-761 amino acids), 

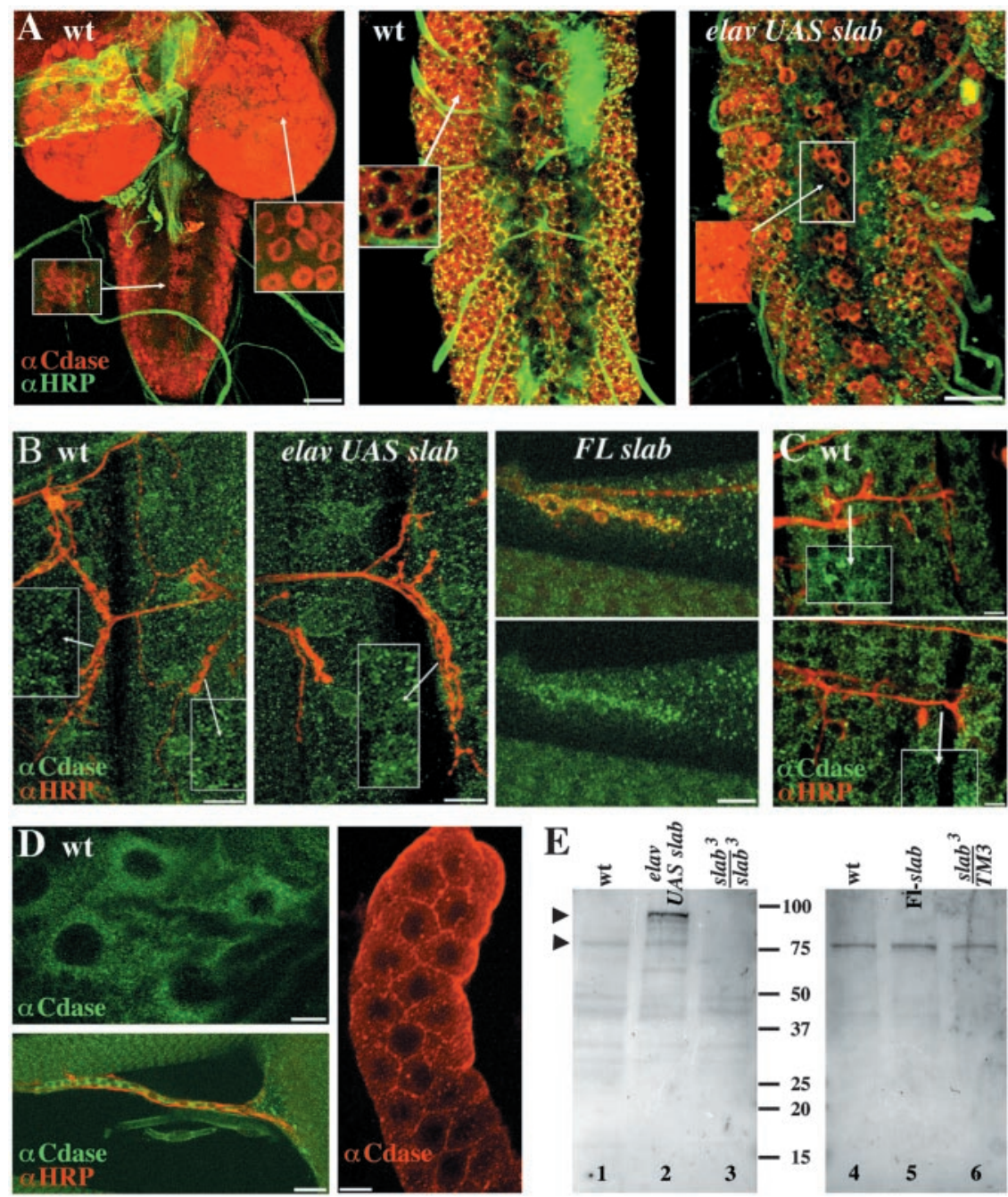

Figure 2. Expression of slab in embryonic, larval, and adult neuronal and non-neuronal tissues. $A-D$, Larval and embryonic preparations stained with antibodies against SLAB Cdase and HRP, showing SLAB is enriched in central neurons. $A$, Left, Wild-type (wt) larval ventral ganglion and central brain lobes, showing prominent SLAB staining (red) within neuronal cell bodies and large central neuroblasts but weaker staining in central and peripheral neuronal processes. Insets at $2 \times$ greater magnification show cytoplasmic staining in the indicated regions. Middle, Wild-type embryonic ventral ganglion, showing similar cellular localization of $S L A B$ (red) within neuronal cell bodies; the inset shows several cells at $3 \times$ greater magnification. Embryonic central brain cells (data not shown) also displayed strong SLAB expression similar to that in larvae. Right, Neuronal UAS-slab overexpression in the embryonic ventral ganglion (elav GAL4; UAS-slab). Confocal gain was substantially reduced to illustrate the cellular staining pattern of SLAB (red). The inset image at left shows the fluorescence of the boxed region captured at the wild-type (center) confocal gain settings for comparison. HRP in all panels is shown in green. Scale bars: left, $40 \mu \mathrm{m}$; middle and right, $20 \mu \mathrm{m}$. $B$, Anti-SLAB (green) and anti-HRP (red) double-staining at larval NMJs. Genotypes shown are wild type (left), elav GAL4 UAS slab neuronal overexpression line (middle), and transgenic Fl-slab line with two additional copies of full-length genomic slab (right). SLAB appears to be expressed in muscle cells but not detectably enriched at the majority of synapses; the insets show SLAB staining alone at $2 \times$ greater magnification for a region of the NMJ indicated by arrows. SLAB antibody staining is detectably enriched at a subset of NMJs in Fl-slab larvae (bottom). Scale bars: left and middle, $20 \mu \mathrm{m}$; right, $10 \mu \mathrm{m}$. C, Embryonic NMJs in wild-type preparations stained for SLAB (green) and HRP (red). The insets show SLAB staining alone for the indicated NMJ regions. Scale bar, $5 \mu \mathrm{m}$. D, SLAB cellular expression in other tissues, including epidermis (green; top left), twist cells (green; bottom left), and salivary gland (red; right). $E_{\text {, }}$ Western analysis of SLAB expression in embryos and adult flies. Extracts from 60 embryos (left; lanes 1-3) or two adult heads (right; lanes $4-6$ ) of the respective genotypes were probed with SLAB antibody. The predicted size of SLAB protein is $78.2 \mathrm{kDa}$ and $\sim 76 \mathrm{kDa}$ with and without signal sequence, respectively. $A$ band of expected size (bottom arrowhead) is present in wild-type adult heads and embryos. The SLAB band is markedly increased in intensity when UAS slab-GFP expression is driven by elav GAL4 (lane 2; SLAB-GFP protein size is increased to $\sim 95 \mathrm{kDa}$ ) and absent in homozygous slab ${ }^{3} / \mathrm{slab}^{3}$ mutants. In adults, the SLAB level relative to wild type is predictably increased by transgenic overexpression (Fl-slab) and decreased in $\mathrm{slab}^{3} / \mathrm{TM} 3$ heterozygotes.

which share $23-39 \%$ amino acid homology and function predominantly in alkaline/neutral environments (Fig. 1D). The slab gene encodes a long Cdase that functions predominantly as an alkaline/neutral form in vitro but reportedly also has significant
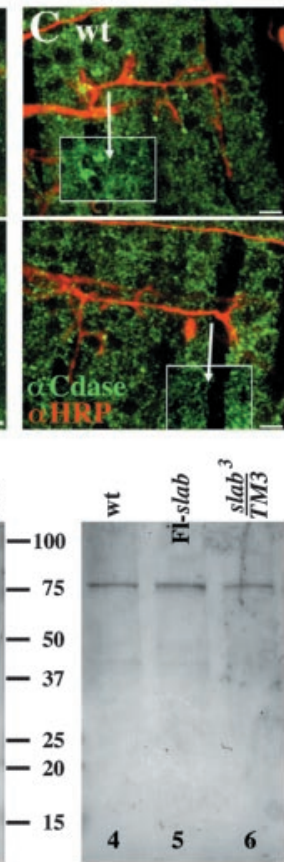

activity at acidic $\mathrm{pH}$ (Yoshimura et al., 2002). The valine altered by the $s l a b^{1}$ mutation is a highly conserved residue in human, mouse, Drosophila, and plant Cdases (Fig. 1C). Other Cdase classes consist of "short" acidic and alkaline forms (264-395 amino acids) (Fig. 1D), which lack homology or common motifs between members of other short or long classes. In Drosophila, the only additional Cdase is a short alkaline form encoded by the brainwashing gene (Boquet et al., 2000).

To confirm that lethality is attributable to mutations in $s l a b$, we rescued lethality with a slab construct consisting of a fulllength slab genomic coding sequence including 3553 bases of endogenous upstream regulatory sequence (Fl-slab). A single copy of the Fl-slab transgene in slab ${ }^{1} /$ slab $^{2}$ and slab $^{1} /$ slab $^{3}$ mutants is sufficient to confer adult viability at percentages near expected for full rescue. In addition, the genomic slab coding sequence was cloned into a pUAS-T vector, and this construct transformed into Drosophila. A single copy of UAS-slab expressed under the control of either a ubiquitous (UH1GAL4) (Wodarz et al., 1995) or a nervous system-specific GAL4 (elav-GAL4) (Luo et al., 1994) driver is sufficient to fully rescue $s l a b^{1} / s l a b^{2}$ flies to adulthood. In contrast, slab expression targeted to muscle using a myosin heavy chain GAL4 fails to rescue $s l a b^{1} / s l a b^{2}$ embryonic lethality. These studies demonstrate that mutation of the Cdase encoded by slab is the sole cause of mutant lethality and demonstrate an essential requirement for slab Cdase within the nervous system.

\section{Expression of SLAB Cdase}

Slab Cdase expressed-sequence tags are present in libraries from Drosophila embryo, larva, pupa, and adult heads as well as from Schneider cells (Rubin et al., 2000), indicating that SLAB is expressed at all stages of the Drosophila life cycle. We assessed SLAB protein expression in embryos, larvae, and adult flies using antibodies including two polyclonal antibodies raised against either the $\mathrm{N}$ terminus (Ab11.3) or C terminus half (Ab10.3) of the protein and three mouse monoclonal antibodies raised against the entire protein. The predicted size of the SLAB protein including signal sequence is $78.2 \mathrm{kDa}$. In Western blots of adult whole fly or head extracts, all antibodies appear highly specific and recognize a prominent band of $\sim 80 \mathrm{kDa}$ (Fig. 2E). Ab10.3 also recognizes a second band of $\sim 130 \mathrm{kDa}$ that may represent a modified/glycosylated adult form of Cdase (data not shown). Immunoreactive band intensity is detectably increased over control in Fl-slab flies with two extra 
copies of $s$ lab and decreased in $s l a b^{3} /$ TM3 heterozygotes with a single copy (Fig. 2E). Anti-SLAB signal is greatly increased when UAS slab expression is driven by a ubiquitous or neuronalspecific GAL4 driver (data not shown). Western blots of wildtype embryonic extracts (30-60 embryos) revealed the expected $\sim 80 \mathrm{kDa}$ SLAB band. The SLAB signal is strongly increased by neuronally driven expression of SLAB-GFP and cannot be detected in $s l a b^{3} /$ slab $^{3}$ mutants, indicating that this allele represents a protein null, as predicted (Fig. $2 E$ ).

The SLAB protein is strongly enriched in neurons, including central neurons in the brain and ventral nerve cord of embryos and larvae (Fig. 2A). The protein is clearly concentrated within the cytoplasm of neuronal cell bodies and weakly expressed or absent from neuronal processes within the central neuropil and in peripheral axons (Fig. 2A). Neuronally driven slab expression greatly increases the staining intensity in central neurons, but the cytoplasmic localization remains similar to that of endogenous SLAB in wild-type neurons (Fig. $2 A$, middle and right). SLAB protein is also evident at a lower level in muscle cells (Fig. $2 B, C$ ). In Fl-slab preparations, a few NMJs exhibit clearly enriched synaptic SLAB localization (Fig. $2 B$, right). However, SLAB staining is not detectably enriched at most NMJs, even when neuronally overexpressed (Fig. $2 B$, left and middle). We also examined a SLAB-GFP protein driven by neuronal elav-GAL4 (data not shown). Consistent with antibody staining results, neuronally expressed SLAB-GFP is present at high levels within neuronal cell bodies and some central axons. SLAB-GFP also appears cytosolic, and its cellular localization is indistinguishable from the intracellular pattern of anti-SLAB staining. The SLAB protein is widely expressed and also present in other tissues in situ, including epidermis, salivary gland, and adult muscle precursor (twist) cells (Broadie and Bate, 1991) (Fig. 2D). In these cells, as in neurons, the SLAB protein appears cytoplasmic with no clear subcellular localization.

\section{Null slab mutants show no detectable increase in cell death} Ceramide derivatives are known signaling molecules in programmed cell death and induce apoptosis in some cell lines (Merrill et al., 1997; Dbaibo and Hannun, 1998; Spiegel et al., 1998; Acharya et al., 2003). During embryogenesis, apoptosis is a normal process for programmed removal of unneeded cells and sculpting of tissues (Abrams et al., 1993). In flies, increased embryonic expression of the death-inducing signal Reaper triggers ceramide generation and activation of caspases, effectors of cell death (Pronk et al., 1996; Bose et al., 1998). A block of Sph-1phosphate hydroylsis in sply mutants results in increased embryonic apoptosis (Herr et al., 2003). The Reaper and ceramide pathway is modulated in part by stress-activated protein kinase/Jun kinase, a target of ceramide signaling (Dbaibo and Hannun, 1998). To address the possibility that ceramide-mediated cell death contributes to the mutant phenotype, we assayed apoptosis in slab null mutant $\left(s l a b^{3} / s l a b^{3}\right)$ and wild-type embryos.

Apoptotic profiles were quantitatively examined in mid-stage embryos (stage 16; 13 hr AEL) by comparison of numbers of acridine orange-stained cells in confocal sections. There was no evidence for increased cell death in slab mutant embryos; $532 \pm$ 50 and $492 \pm 126$ acridine orange-stained cells were counted in wild-type and slab preparations, respectively (mean $\pm \mathrm{SD} ; n=5$ for both genotypes). The absence of detectably increased cell death in slab Cdase mutants is likely attributable to cellular checkpoints preventing unwanted cell death. For example, Drosophila caspase activity is inhibited by binding of DIAP1 (inhibitor of apoptosis 1); this inhibition is relieved by several proteins including Reaper, which thus regulates apoptosis both upstream and downstream of ceramide generation (Holley et al., 2002). Additionally, Bcl-2 can protect mammalian cells from ceramideinduced apoptosis (Dbaibo and Hannun, 1998). The Drosophila homologs buffy and death executioner Bcl-2 likely perform similar protective functions in the fly. We conclude that slab mutant lethality is unlikely to be a result of decreased neuronal viability or cell maintenance.

\section{SLAB Cdase regulates synaptic sphingolipid level and distribution}

Drosophila raft lipid content has been investigated in extracted embryonic membranes (Rietveld et al., 1999); however, no known specific probes or markers exist for endogenous Drosophila sphingolipids or raft domains. Fluorescent sphingolipid analogs have been widely used to study sphingolipid trafficking and metabolism in mammalian in vitro systems (Putz and Schwarzmann, 1995; Pagano and Chen, 1998; Pagano et al., 2000b). Ceramide analogs with a fluorophore attached to a shortened fatty acid chain are rapidly incorporated into the PM, internalized and concentrated in sphingolipid-rich domains within the Golgi, and subsequently metabolized to higher fluorescent sphingolipids and glycosphingolipids and trafficked via vesicles back to the surface membrane (Lipsky and Pagano, 1985; Pagano and Sleight, 1985; Pagano et al., 1989, 1991, 2000b; Putz and Schwarzmann, 1995).

We assessed the distribution and cellular trafficking of BODIPY Fl $\mathrm{C}_{5}$-ceramide $\left(\mathrm{C}_{5}\right.$-Cer) (Pagano and Chen, 1998; Pagano et al., $2000 \mathrm{~b}$ ) at living NMJ synaptic membranes and asked whether localization of fluorescent ceramide over a 1-2 hr time course is dependent on the SLAB expression level. Dissected third instar larvae and mature embryos (22-24 AEL) were incubated in $\mathrm{C}_{5}$-Cer $(5-10 \mu \mathrm{M})$ in Schneider's medium for 30-45 min, washed at room temperature for $1-2 \mathrm{hr}$ to remove excess surface labeling, and imaged on the confocal microscope. Significant fluorescence is present in many cells, consistent with expected surface incorporation and internal trafficking of the lipid. In muscle cells, for example, $\mathrm{C}_{5}$-Cer is diffusely incorporated throughout the PM as well as accumulated within internal organelle membranes, including the ER membrane surrounding nuclei (Fig. 3 and data not shown). Little or no fluorescence is found in the CNS and ventral ganglion, presumably because of the glial sheath barrier.

$\mathrm{C}_{5}$-Cer is concentrated to NMJ synapses, most prominently at large ( $>3 \mu \mathrm{m}$ diameter) "type Ib" synaptic boutons, which are surrounded by multi-layered postsynaptic subsynaptic reticular (SSR) membranes (Fig. $3 A, B, D$ ). As confirmation of synaptic localization, we compared $\mathrm{C}_{5}$-Cer distribution with that of antiDLG, a synaptic marker associated primarily with the SSR of type I boutons. After labeling and imaging of $\mathrm{C}_{5}$-Cer at living NMJs, preparations were fixed and stained with anti-DLG, and the identical DLG-stained NMJs were then re-imaged, confirming that $\mathrm{C}_{5}$-Cer fluorescence is consistently enriched at synaptic boutons (Fig. 3A). In addition, $\mathrm{C}_{5}$-Cer is strongly accumulated at many small type II boutons that do not have associated postsynaptic SSR membranes (Fig. $3 B$ ). $\mathrm{C}_{5}$-Cer is also clearly concentrated at embryonic synaptic boutons before SSR development (Fig. 3D). These results provide evidence that ceramide, the substrate for $\mathrm{SLAB}$, is characteristically enriched and localized in embryonic synapses at the time of essential requirement (see below) as well as in mature larval stages. To address whether altered SLAB expression modifies the synaptic sphingolipid environment in vivo, we compared and quantified $\mathrm{C}_{5}$-Cer levels at type I larval boutons between transgenic Fl-slab larvae and $s l a b^{3} / T M 3$ heterozgotes (Fig. 3D,E). Mean synaptic fluorescence incorporated at Fl-slab 

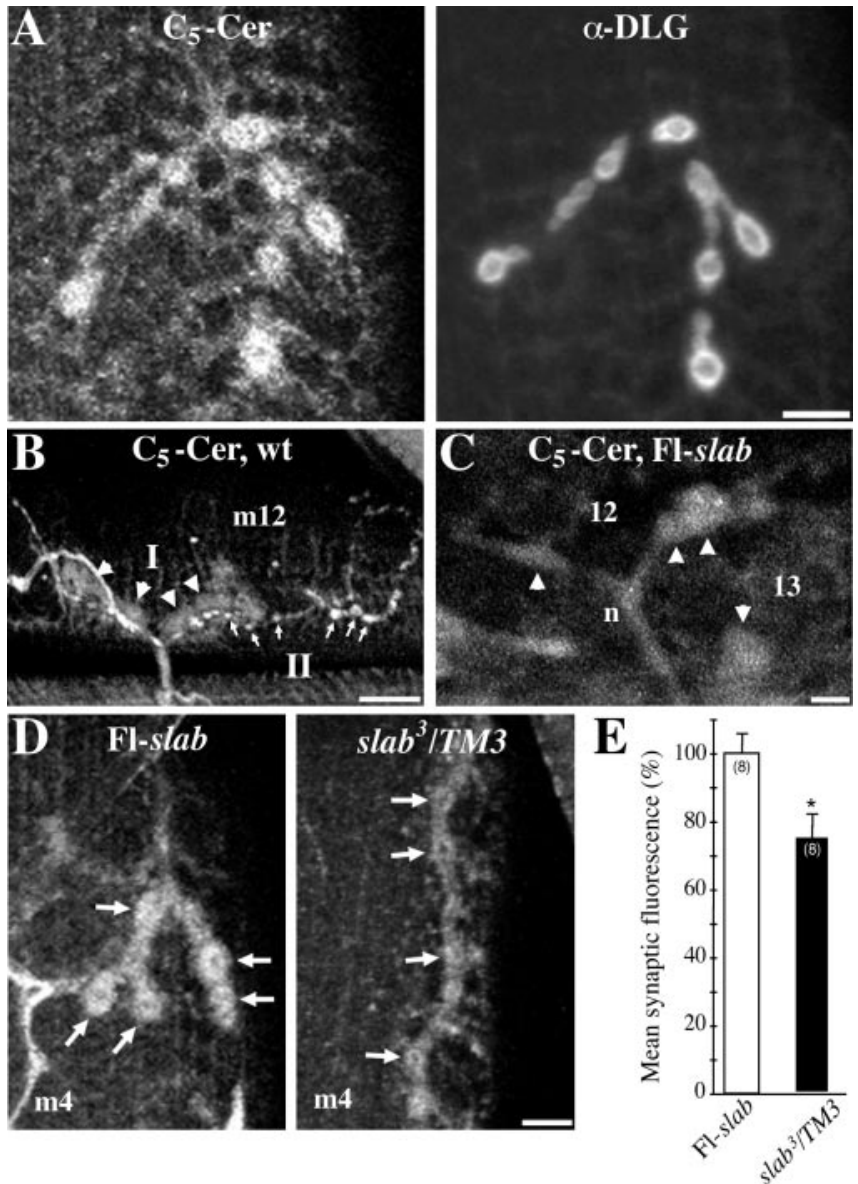

Figure 3. Fluorescent $\mathrm{C}_{5}$-ceramide is trafficked to $\mathrm{NMJ}$ membranes, and synaptic accumulation is dependent on the level of SLAB expression. $A$, Left, The fluorescent ceramide analog BODIPY FL $\mathrm{C}_{5}$-ceramide $\left(\mathrm{C}_{5}\right.$-Cer) is incorporated into neuronal and muscle membranes and strongly localized to living larval NMJ boutons within $1-2 \mathrm{hr}$ after a 30 min incubation. Shown is the NMJ at muscle 4 in a living larval preparation. Right, The identical NMJ imaged after fixation and staining with an antibody against DLG, which localizes to type lb synaptic boutons. Scale bar, $10 \mu \mathrm{m}$. B, Wild-type NMJ after incubation in $\mathrm{C}_{5}$-Cer, showing fluorescence concentrated at both large type Ib boutons (I; arrowheads) and small type II boutons (II; arrows). Labeling of the presynaptic axonal membrane and type II boutons lacking postsynaptic SSR indicates that sphingolipid-enriched environments are present presynaptically as well as postsynaptically. Scale bar, $20 \mu \mathrm{m}$. C, $C_{5}$-Cer accumulated at embryonic synapses (NMJ 12/13, Fl-slab embryo). Synaptic boutons (arrowhead) and presynaptic nerve ( $\mathrm{n}$ ) are indicated. Scale bar, $2 \mu \mathrm{m} . D_{1}$, Comparison of $\mathrm{NMJ}_{5}$-Cer fluorescence in Fl-slab larva with two additional copies of genomic slab (left), with that in slab ${ }^{3} / \mathrm{TM} 3$ heterozygote with a single copy of slab. Both images shown are Z-series of $5 \mu \mathrm{m}$ total thickness for comparison of synaptic accumulation and are representative of quantified fluorescence differences in eight paired experiments. Scale bar, $10 \mu \mathrm{m}$. E, Quantified mean NMJ synaptic $\mathrm{C}_{5}$-Cer fluorescence (muscle 4) in slab ${ }^{3} / \mathrm{TM}$ larvae (filled bar), normalized to that in Fl-slab larvae in eight paired experiments. * ${ }^{*}$ ignificant reduction in mean fluorescence relative to Fl-slab $(p<0.02)$.

NMJs is significantly increased compared with $s l a b^{3} / T M 3$ NMJs $\left(p<0.02\right.$ ) (Fig. 3E). Because $\mathrm{C}_{5}$-Cer was acutely applied and imaged (within $\sim 2 \mathrm{hr}$ ), this result indicates that the localized endogenous synaptic sphingolipid environment is regulated by SLAB function and altered by the level of SLAB activity.

\section{Essential requirement for SLAB Cdase in neuronal function}

The slab mutant lethal phenotype is highly penetrant in all allelic combinations of slab mutants $\left(\right.$ slab $^{1}$, slab $^{2}$, slab $^{3}$ homozygotes and transheterozygote combinations) (Fig. $1 B$ ), with $\sim 99 \%$ of mutant animals dying either during or very shortly after hatching. Mutant embryos appear anatomically and morphologically
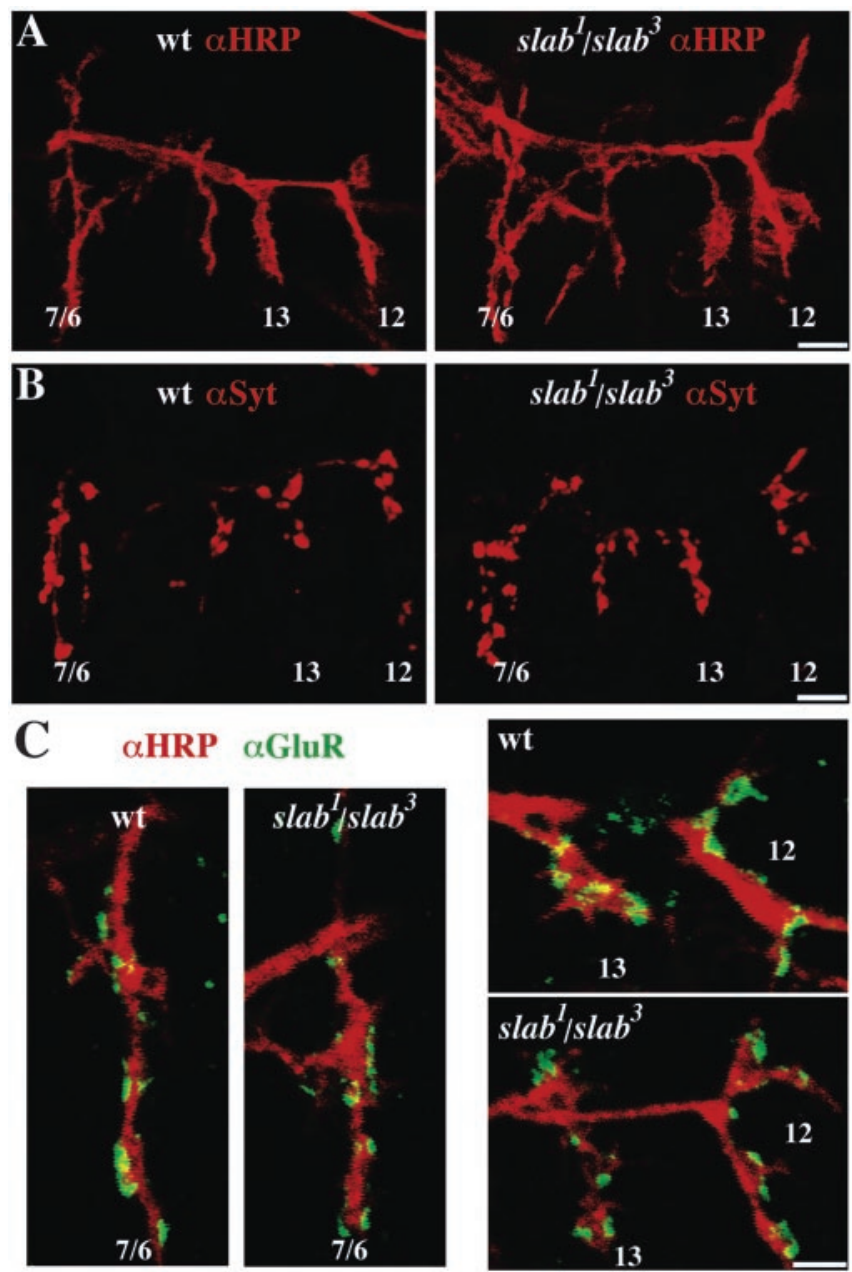

Figure 4. Null slab mutant NMJs have normal presynaptic terminal morphology and postsynaptic glutamate receptor localization. $A$, NMJ terminals on identified muscles $(7,6,13$, 12) within a single ventral hemisegment in mature (20-22 hr AEL) wild-type (wt; left) and $\mathrm{slab}^{1} / \mathrm{slab}^{3}$ null mutant (right) embryos, visualized with anti-HRP antibody staining. These NMJ terminals are the subject of synaptic electrophysiology, SV dye imaging, and ultrastructural studies (see Figs. 5- 8). Scale bar, $5 \mu \mathrm{m}$. B, Localization and distribution of anti-synaptogmin I, a SV protein, showing normal morphology and differentiation of NMJ synaptic boutons in slab null mutants. C, NMJs of wt and slab null mutants double-stained against HRP ( $\alpha$ HRP; red) and the GluRIIIA postsynaptic glutamate receptor ( $\alpha$ GluR; green). The left pair of panels shows NMJ 6/7; the right pair shows NMJ 12/13. GluR puncta are closely opposed to presynaptic boutons. GluR puncta size and appearance are indistinguishable between control and slab NMJs, and slab GluR functional responses show no postsynaptic impairment (see Fig. 50). Scale bar, $2.5 \mu \mathrm{m}$.

normal, including a normally segmented epidermis, tracheal system, gut, nervous system, and musculature (data not shown and Fig. 4). Mutants move spontaneously and in response to touch before hatching, although both spontaneous and evoked movement are less vigorous than in control embryos, initiate hatching (21-22 hr AEL at $25^{\circ} \mathrm{C}$ ), and protrude their heads from the egg case. The majority of mutant animals exhibit only weak head movements for a few minutes and fail to move further. A minority briefly generate peristaltic body contractions sufficient to emerge partly or completely from the egg case; a few succeed in hatching completely but rarely move more than a few body lengths. When moved to an aqueous environment before or immediately after hatching, most mutant animals maintain spontaneous and touch-evoked movements for 1-2 hr. However, persistence of coordinated whole-body peristaltic locomotion behavior typical of normal animals is never observed. Heterozy- 
gous slab $/ T M 3$ balanced siblings display wild-type movement and behavior as well as physiological transmission properties (see below).

Examination of the nervous system and somatic muscles with the light microscope reveals no obvious anatomical defects in slab mutants. NMJ morphology and differentiation was examined at a confocal level with presynaptic and postsynaptic antibody markers to investigate possible defects in synaptic morphogenesis or molecular differentiation. In mature slab mutant embryos (22-24 hr AEL), NMJs are correctly and stereotypically formed, with presynaptic terminal elaboration, branching, and synaptic bouton formation indistinguishable from control synapses (Fig. $4 A, B$; see also Fig. 7). We measured areas of NMJ presynaptic terminals in anti-HRP-stained embryos and confirmed that NMJ arbor size is not significantly altered in slab mutants $(p>0.5$; sla $^{1} /$ slab $^{1}$ vs wild type; muscles 12 and $13 \mathrm{NMJs;} n=5$ animals). Mutant NMJs also exhibited normal levels and distribution of antibody staining against SV proteins such as Syt1 (Fig. 4B). Likewise, staining against postsynaptic glutamate receptors (GluRs; GluRIIA subunit) (Petersen et al., 1997; Saitoe et al., 1997) reveals GluR punctae closely opposed to presynaptic boutons at both slab and control NMJs (Fig. 4C). GluR cluster appearance and number do not differ detectably between control and mutant NMJs; furthermore, direct assays of GluR receptor function show no impairment in slab mutants (see below), suggesting that the postsynaptic receptor field is normally formed and aggregated. These studies indicate that the severe movement impairment of slab mutants is likely associated with impaired neuromuscular function.

\section{Impaired neurotransmission at slab mutant NMJs is attributable to a presynaptic defect}

Electrophysiological assays were performed in slab mutants at mature embryonic stages (20-21 hr AEL) and in larvae immediately after hatching (22-23 hr AEL). Direct electrical stimulation of the muscle or application of glutamate to the postsynaptic muscle membrane results in visible muscle contraction in slab mutants similar to that observed for control (wild-type or slab/ TM3 heterozygotes) animals, indicating that mutants retain muscle function. In control animals, electrical stimulation of the ventral ganglion or peripheral motor nerves evokes strong segmental muscle contraction and usually patterned waves of peristaltic muscular activity. In contrast, such stimulation evokes only weak, local muscle contraction in slab mutants, consistent with behavioral movement defects indicating a specific defect in the nervous system or NMJ synaptic transmission.

Whole-cell patch-clamp recordings were made from voltageclamped $(-60 \mathrm{mV})$ embryonic muscle $(\mathrm{m} 6)$ (Broadie and Bate, 1993). Spontaneous EJC events are recorded at nearly all slab mutant NMJs, indicating the presence of functional glutamatergic transmission. However, EJCs evoked by direct electrical stimulation of the motor nerve are substantially impaired at slab NMJs. At control NMJs, nerve-evoked EJCs are robust and reproducible, with mean amplitudes of $\sim 1000 \mathrm{pA}(963 \pm 81 \mathrm{pA}$, wild type, $n=4 ; 987 \pm 59 \mathrm{pA}$, slab $/$ TM3, $n=7$ ) at basal stimulation frequencies of $0.2-0.5 \mathrm{~Hz}$ (Fig. $5 \mathrm{~A}$ ). In contrast, slab mutant NMJs display significantly reduced, low-fidelity evoked transmission. Evoked EJC amplitudes display considerable variation and are reduced, on average, by $50-70 \%$ overall in different slab alleles $\left[\right.$ slab $^{1} /$ slab $^{1}(327 \pm 92 \mathrm{pA} ; n=7 ; p<0.01)$ vs slab ${ }^{1} /$ TM3; slab $^{1} /$ slab $^{2}(490 \pm 59 \mathrm{pA} ; n=6 ; p<0.01)$ vs slab $^{1} / \mathrm{TM} 3$ ] (Fig. $5 A)$. The incidence of transmission failures and near-failures at slab NMJs is progressively increased at higher $(5-10 \mathrm{~Hz})$ stimu-
$\mathbf{A}$

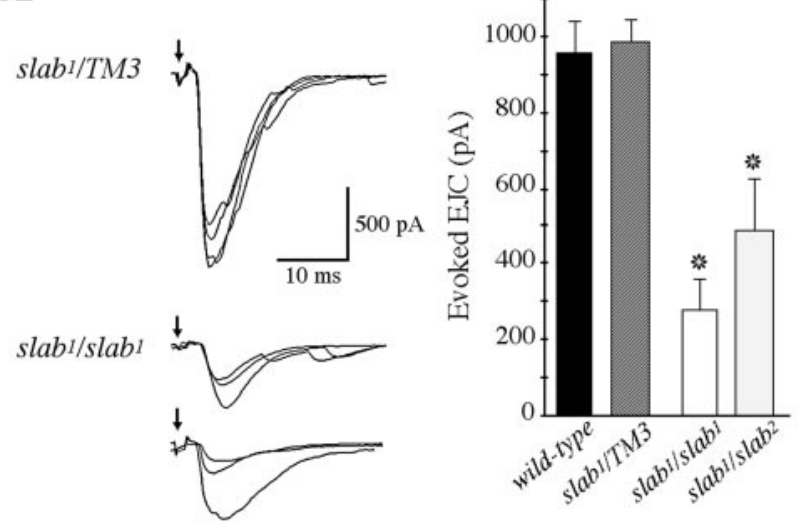

B

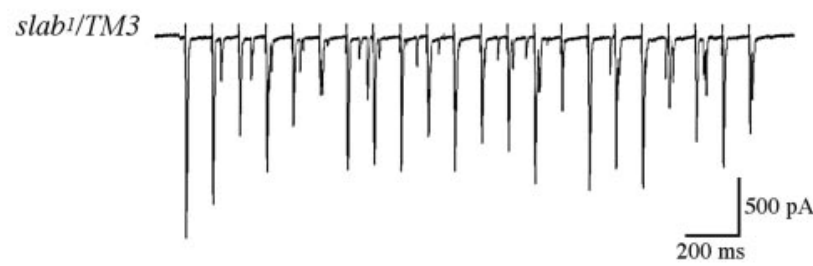

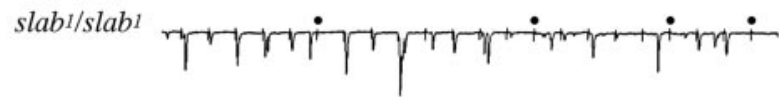

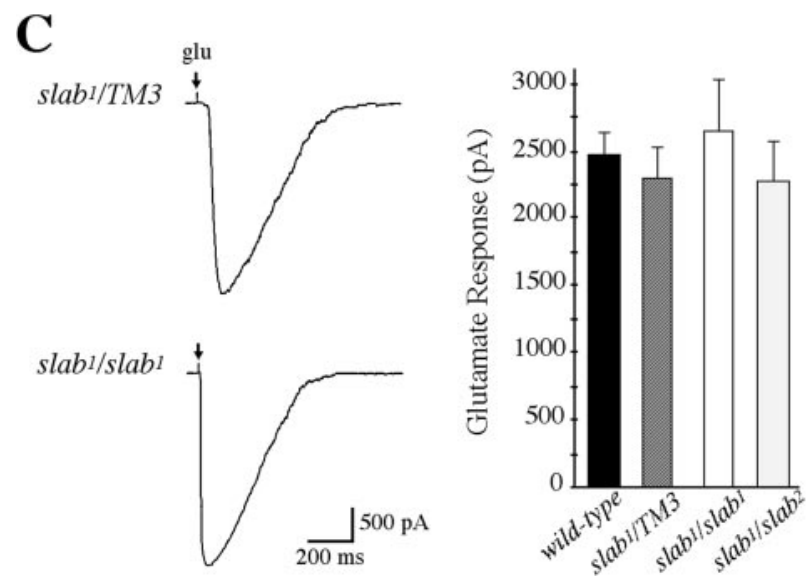

Figure 5. Impaired neurotransmission at slab mutant NMJs is attributable to a presynaptic defect. $A$, Representative nerve-evoked (arrow) EJCs from control (slab $/$ TM3; top traces) and homozygous slab ${ }^{1}$ mutant NMJs (bottom traces; examples from 2 animals are shown). In controls, EJCs are robust $(\sim 1 \mathrm{nA})$ and reproducible. In slab mutants, EJC amplitude and fidelity are reduced and include transmission failures and near-failures. The mean EJC amplitudes are plotted at right for controls (wild-type, slab $/ T M 3$ ), slab $/$ slab $^{7}$ mutants ( ${ }^{*} p<0.01$ vs slab $/ T M 3$ ) and $s l a b^{1} / s l a b^{2}$ mutants ( ${ }^{*} p<0.01$ vs slab $\left.{ }^{1} / T M 3\right)$. The two slab alleles are not significantly different ( $p>0.13) . B$, Higher-frequency $(10 \mathrm{~Hz})$ evoked EJC trains illustrate well maintained transmission under elevated demand at control NMJs. In contrast, slab mutant NMJs show severely reduced amplitudes and erratic transmission, including failures (marked with symbols). C, Postsynaptic current responses elicited by direct iontophoretic application of glutamate. Representative traces (left) show glutamate-gated currents at control and slab NMJs. No difference in mean glutamate current amplitudes (plotted at right) is observed between controls (wild-type, slab $\left.{ }^{1} / T M 3\right)$ and slab mutant $\left(s l a b^{1} / s l a b^{7}\right.$, slab $/$ slab $\left.^{2}\right)$ alleles.

lation frequencies, suggesting a reduced efficacy of presynaptic transmitter release in response to evoked nerve activity (Fig. 5B). The decreased mutant EJC amplitudes are not attributable to a failure in evoked action potential induction or propagation, be- 
cause this direct nerve stimulation technique is readily able to support release by electronic presynaptic depolarization (Broadie and Bate, 1993; Auld et al., 1995). Thus, although a level of endogenously generated transmission and weakened evoked transmission persists at slab mutant synapses, normal coupling of transmission to presynaptic depolarization is greatly reduced, with transmission fidelity increasingly compromised at higher levels of activity.

As noted above, direct visualization of postsynaptic GluR fields at slab mutant NMJs indicates that receptors appear localized and clustered similarly to wild type. We directly tested GluR function by recording postsynaptic current responses evoked by brief $(2-10 \mathrm{msec})$ iontophoretic application of glutamate directly to the muscle $6 / 7$ synapse (Fig. $5 C$ ). Glutamate application produced visible muscle contraction in all recorded slab preparations, and robust glutamate current responses were recorded at all slab mutant NMJs, with current amplitudes indistinguishable from controls [e.g., $2516 \pm 381 \mathrm{pA}\left(\right.$ slab $^{1} /$ slab $\left.^{1}\right)$ vs $2292 \pm 235 \mathrm{pA}$ $\left(s l a b^{1} / T M 3\right) ; p>0.3$ ] (Fig. 5C). Strong postsynaptic function was present regardless of the level of presynaptic function suggested by endogenous transmission present at individual NMJs. These results indicate that the postsynaptic GluR field is normally differentiated and functional and that slab mutants are impaired specifically in presynaptic function.

\section{Reduction in fusion of readily releasable SVs at slab synapses}

The preceding analyses demonstrate a defect in the coupling of presynaptic depolarization to neurotransmitter release in slab mutant synapses. Several potential mechanisms, including a reduced presynaptic $\mathrm{Ca}^{2+}$ signal, a reduction in the readily releasable pool (RRP) of SVs, or an impairment in the SV fusion mechanism, could explain the release defect. To differentiate between these possibilities, we next assayed fusion evoked by HO saline, which specifically triggers the release of the RRP independently of $\mathrm{Ca}^{2+}$ influx (Rosenmund and Stevens, 1996). Whole-cell postsynaptic currents were recorded in response to brief (2 sec) focal applications of $\mathrm{HO}$ saline to the m6/7 NMJ (Aravamudan et al., 1999; Fergestad et al., 2001).

At control NMJs, HO saline evokes a well defined, sustained episode of vesicular fusion events (Fig. 6 A). HO-evoked current responses were quantified by measuring both total synaptic charge as well as the number of discrete fusion events (number of current peaks) (Fig. 6B) over the 4 sec period after the onset of the application. For both parameters, responses at wild-type and slab/TM3 control NMJs are indistinguishable (slab/TM3: $76.4 \pm$ 7 pAwS mean current area; $83 \pm 6$ current peaks; $n=12$ ). In contrast, $\mathrm{HO}$ responses at slab ${ }^{1} /$ slab $^{1}$ mutant NMJs are reduced by $70 \%(22.8 \pm 5.5$ pAwS; $22 \pm 6$ current peaks; $n=10)$. Likewise, $s l a b^{1} /$ slab $^{2}$ and $s l a b^{1} /$ slab $^{3}$ allelic combinations were also significantly reduced in both response parameters (Fig. 6A,B). A straightforward interpretation of these results is that slab synapses have a reduced pool of readily releasable SVs. If the defect arises from a loss of docked SVs at the presynaptic membrane, this should be apparent in ultrastructural analysis (see below). Alternatively, the loss of the RRP in slab mutants may indicate a direct impairment of the SV fusion mechanism itself.

\section{FM1-43 dye assays indicate a smaller cycling pool of SVs at slab mutant synapses}

A loss of readily releasable SVs at slab mutant synapses may be a result of impairments in vesicle biogenesis, endocytosis, or trafficking of SV pools. Assays of FM1-43 dye uptake and release in living terminals provide an optical assay of activity-dependent SV
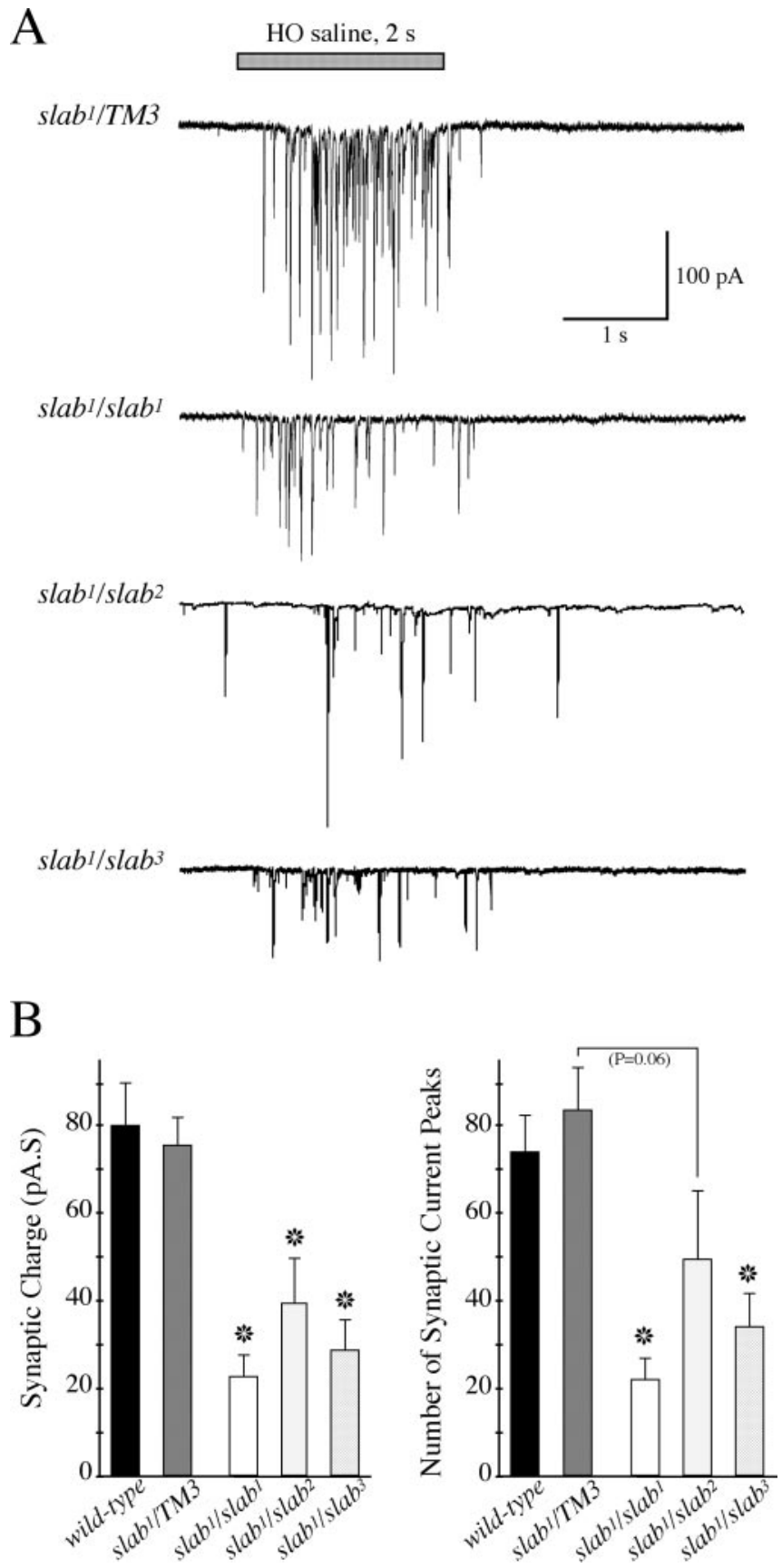

Figure 6. Presynaptic vesicle fusion evoked by $\mathrm{HO}$ saline is reduced at slab mutant synapses. $A$, Representative responses to 2 sec focal applications of $\mathrm{HO}$ saline ( $1175 \mathrm{mosM}$; stippled bar) to

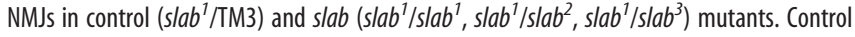
responses typically consist of a dense burst of SV fusion events, representing release of the docked SV population. Mutant responses ranged from slight reduction in fusion compared with controls to nearly complete absence of response; overall mean reduction is significant for each slab allelic combination. $B$, Quantification of synaptic charge (left) and number of fusion events (synaptic current peaks; right) evoked by $\mathrm{HO}$ saline in controls (wild-type, slab ${ }^{1} / \mathrm{TM} 3$ ) and slab mutants. Asterisks indicate statistical significance $(p<0.05)$ compared with slab ${ }^{1} / \mathrm{TM} 3$ control.

pool size and trafficking dynamics to functionally test these possibilities (Fergestad and Broadie, 2001). NMJ synapses in slab mutant and control preparations were loaded with FM1-43 during a 2.5 min depolarization with high $\mathrm{K}^{+}$(90 mM)-containing saline and identified NMJ terminals imaged on the confocal microscope. Terminals were then destained with a second high $\mathrm{K}^{+}$ 
depolarization of varying duration (30 sec to $2.5 \mathrm{~min}$ ) and re-imaged. These conditions reliably and consistently load and unload the "endo/exo" cycling SV pool at embryonic NMJs (Fergestad and Broadie, 2001), similar to studies at larval NMJs (Kuromi and Kidokoro, 1998, 2000).

In loaded terminals, dye fluorescence is typically localized to the periphery of larger ( $\geq 2 \mu \mathrm{m}$ diameter) embryonic boutons (Fig. $7 A, B)$, showing that SVs are concentrated in cortical regions underlying the presynaptic $\mathrm{PM}$, as in mature larval boutons. Dye is also incorporated throughout the interior of some boutons, in particular smaller boutons (Fig. 7A, B), although usually at less intensity, corresponding to a lower SV density in interior regions. The pattern of dye uptake suggests that most or all of the SV population in embryonic NMJ terminals may participate in active endo/exo cycling, differing from larval NMJs, which contain a large reserve pool that is not cycled under these conditions (Kuromi and Kidokoro, 1999, 2000).

A 2.5 min loading protocol appears to nearly maximally load the cycling SV pool in both controls and slab mutants. The spatial pattern and distribution of loaded dye within normal and slab mutant boutons appears qualitatively similar (Fig. $7 A, B$ ). However, slab terminals display significantly reduced quantified mean loaded fluorescence compared with control values $(69 \pm 4 \%$ of control; $p<0.0001$ ) (Fig. $7 C$ ), indicating a reduction in the size of the actively cycling mutant SV pool. A 1-2.5 min $90 \mathrm{~mm} \mathrm{~K}^{+}$ depolarization effectively destains $\sim 80 \%$ of loaded fluorescence in both control and $\mathrm{mu}-$ tant terminals ( $84 \pm 3 \%$ control vs $77 \pm 5 \%$ slab; $p>0.4$ ), showing that slab synapses are capable of cycling and releasing the smaller SV pool in a similar period of time (Fig. $7 C$ ). No significant difference in the unloaded fractions between control and slab NMJs was measured for $30 \mathrm{sec}, 1 \mathrm{~min}$, or $2.5 \mathrm{~min}$ depolarizing stimuli, suggesting a cycling pool turnover time of $<30 \mathrm{sec}$ when challenged with $90 \mathrm{mM} \mathrm{K}^{+}$. To target possible differences in the rate of SV pool release, we further assayed destaining using more moderate depolarizing conditions. As shown in Figure $7 D$, fractional release with $30 \mathrm{~mm} \mathrm{~K}^{+}$ for either 1 or $2 \mathrm{~min}$ is comparable between slab and wild-type NMJs. Therefore, during sustained high $\mathrm{K}^{+}$-mediated depolarization, slab mutant synapses are capable of trafficking and releasing SVs similarly to control synapses. However, the actively cycling slab SV pool is reduced in size, consistent with a reduced ability to maintain neurotransmitter release during repetitive stimulation.
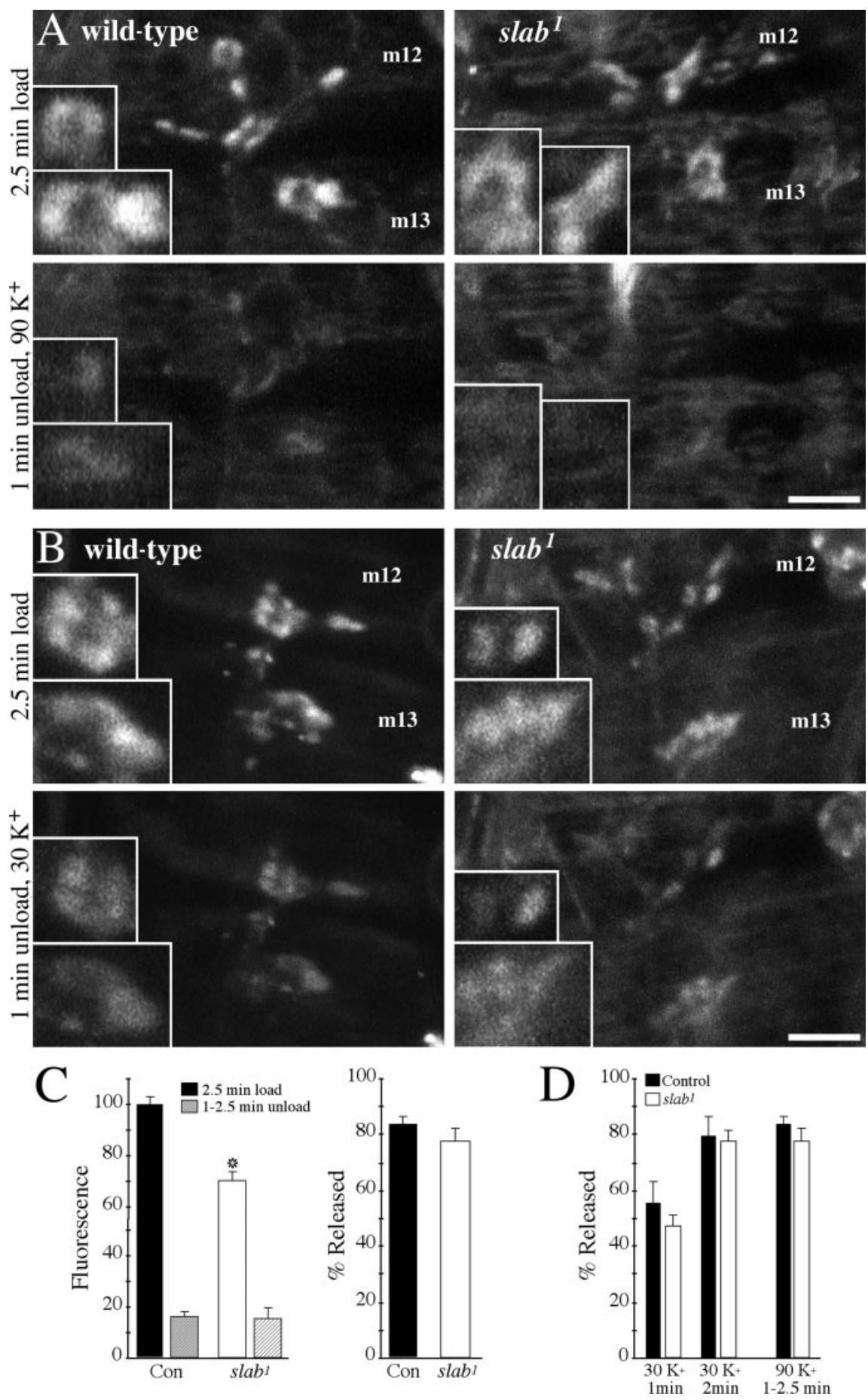

Figure 7. FM1-43 imaging indicates a reduced cycling SV pool size in slab mutant synapses. FM1-43 dye (10 $\mu$ m) was loaded into NMJ boutons with 2.5 min depolarizing stimulation with saline containing $90 \mathrm{~mm} \mathrm{~K}^{+}$. After imaging of loaded fluorescence at identified NMJ 12/13 terminals, dye was unloaded with 1-2.5 min stimulation with 90 or $30 \mathrm{~mm} \mathrm{~K}^{+}$saline to maximally or partially destain the terminal. $A$, Representative images of loaded and unloaded ( $1 \mathrm{~min}, 90 \mathrm{~mm} \mathrm{~K}^{+}$) wild-type control (left) and slab ${ }^{1}$ (right) NMJs. The insets show individual large boutons at each terminal at twofold increased magnification. $B$, Representative images of loaded and partially destained NMJs ( $\left.1 \mathrm{~min}, 30 \mathrm{~mm} \mathrm{~K}^{+}\right)$in wild-type and slab ${ }^{1}$ NMJs. C, Quantified mean fluorescence after loaded (solid bars) and remaining after subsequent destaining with $90 \mathrm{~mm} \mathrm{~K}^{+}$(stippled bars; 1 and 2.5 min unloading experiments are pooled) in wild-type control (Con) and slab ${ }^{1}$ mutants (left). Values are normalized to mean control loaded fluorescence (100\%). Mean dye loading of slab NMJs is significantly reduced ( $\left.{ }^{*} p<0.0001\right)$ compared with control. The right plot shows the percentage of loaded fluorescence released by destaining stimulation (\% Released) for the same experiments. Control and slab mutant $90 \mathrm{~mm} \mathrm{~K}^{+}$percentage unloading shows no significant difference. D, Percentage release of loaded fluorescence by 1 and 2 min stimulation with $30 \mathrm{~mm} \mathrm{~K}^{+}$saline; $90 \mathrm{~mm} \mathrm{~K}^{+}$unloading is shown for comparison (rightmost pair of bars). Unloading for slab mutants is not significantly different from that in controls for each destaining condition, indicating cycling of the mutant SV pool under these conditions is not detectably altered. 


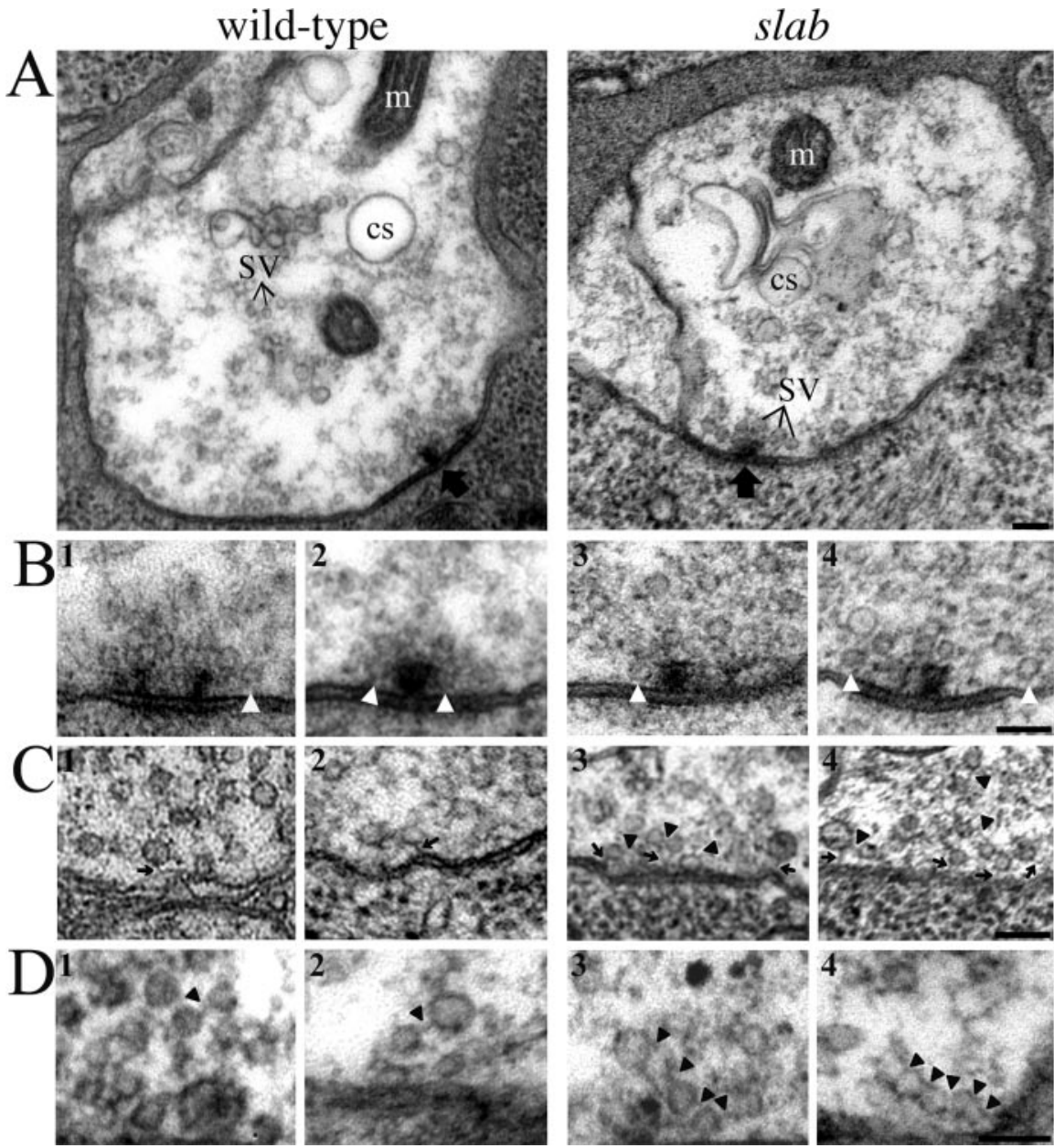

Figure 8. slab mutant terminals have normal clustered and docked RRP but a reduction in nonclustered SVs and an accumulation of linked and tethered RP SVs. Electron micrographs of synaptic ultrastructure at the NMJ of fully developed (20 -22 hr AEL) wild-type control (left; $A-D$ ) and $s l a b^{7} /$ slab $^{3}$ (slab; right; $A-D$ ) mutant embryos. $A$, Representative profiles of wild-type (left) and slab (right) boutons, each displaying an electron-dense presynaptic T-bar and electron-dense PM marking the AZ (large arrowheads). SVs are clustered near the AZ and distributed throughout the bouton interior and periphery. Similar profiles were used for all quantitative analyses. cs, Cisternae; $\mathrm{m}$, mitochondria. Scale bar, $100 \mathrm{~nm}$. B, Detailed images of wild-type $(1,2)$ and slab $(3,4)$ AZ regions, showing the population of SVs clustered near the AZ and docked SVs (arrowheads) at the PM. C, Detailed images illustrating SVs tethered by filamentous structures (arrows) to the non-AZ PM. Linked SVs, defined as those either in direct contact or connected by electron-dense structures, are present among the SV visible in slab panels (3, 4, arrowheads). D, Linked SVs in wild-type and slab terminals; arrowheads indicate linking structures or point of contact between linked SVs. Linked/tethered SVs in slab boutons often appear as arrays or rows aligned near the PM as in panels 3 and 4 . Significantly increased numbers and percentages of linked and tethered SV s are present in slab mutant boutons (see Table 1). Scale bars, $A-D$ (in right panels), $100 \mathrm{~nm}$.

\section{Electron microscopy reveals aberrant SV trafficking in slab mutant synapses}

Ultrastructural analyses of embryonic NMJ terminals were undertaken to assess the hypothesis that loss of SLAB Cdase function alters SV pool size or distribution. Ultrastructural features examined included PM appearance and integrity; bouton crosssectional area; AZ regions, defined by electron-dense PM and synaptic cleft; electron-dense "T-bar" bodies at AZ fusion sites; SV morphology and diameter; number of SVs clustered and docked at the AZ; and number/distributions of SVs within the bouton interior (Fig. 8, Table 1). As in previous studies (Aravamudan et al., 1999; Featherstone et al., 2001), clustered SVs were defined as those within $250 \mathrm{~nm}$ of an AZ, and "docked" SVs as those within $<0.5 \mathrm{SV}$ diameter $(<20 \mathrm{~nm})$ of the AZ membrane in the section containing the most prominent T-bar structure.

In all fundamental respects, synaptic ultrastructural differentiation at the EM level appears unaltered at slab mutant presyn- aptic terminals (Fig. 8A). Membrane integrity is preserved, and terminal organelles, including SVs, maintain normal morphology and size. Mutant terminals exhibit electron-dense AZ membranes and T-bar densities, and SVs clustered at the AZ. The clustered population at the $\mathrm{AZ}$ contains the same number of SVs in control and mutant synaptic profiles (13 \pm 1 SV/AZ for both wild type and slab) (Table 1). Surprisingly, we found that slab AZs also exhibited normal or greater numbers of docked SVs $(2.5 \pm 0.2)$ compared with wild-type AZs $(2.1 \pm 0.2)$ (Table 1). This result indicates that the slab release impairment is not attributable to deficiencies in localizing SVs to release sites or in docking of RRP SVs to the membrane, but rather a downstream defect in SV priming/fusion. One possible explanation for this phenotype would be a disrupted assembly or maintenance the mutant AZ. We therefore quantified the length of mutant $A Z$ membranes and cross-sectional areas of presynaptic T-bar structures, which are known to serve a fusogenic function. There were no significant differences between wild-type and slab mutants in either of these features. Thus, slab mutants must be specifically defective in SV priming/fusion immediately upstream of neurotransmitter release.

In addition, slab mutant terminals exhibit several significant differences in the size and distribution of the internal nonclustered SV pool, suggesting defects in vesicle trafficking. First, although overall SV density throughout the bouton is not significantly reduced, slab boutons have, on average, $\sim 30 \%$ fewer SVs within the nonclustered population removed from the AZ ( $p<0.05$ vs wild type) (Table 1 ). This reduction in SV number closely matches the reduction in mutant FM1-43 uptake, supporting the conclusion that slab synapses have a smaller functional cycling pool. Second, slab boutons contain increased numbers of SVs linked together in multi-vesicle clusters or chains (Fig. 8C,D; Table 1). These vesicles appear either in direct contact or to be connected by electron-dense globular or filamentous structures and are located both within the bouton interior as well as near the PM. Similar SV-SV linkages are present in normal terminals but are increased twofold to threefold in slab boutons. Third, slab mutant boutons exhibit increased numbers of SVs tethered by filamentous structures to the PM. Tethered vesicles resemble docked vesicles but are not localized to AZs and are typically located at a greater distance $(\sim 1$ vesicle diameter $)$ from the membrane. To confirm that tethered SVs were not associated with nearby AZs in adjacent sections, we scored and counted tethered SVs in serial sections in a subset of boutons (two to four sections/ bouton in 32 boutons). This analysis indicated that tethered SVs tended to be localized to PM regions removed from an AZ. The incidence of both linked and tethered SVs is increased by twofold 
Table 1. Summary of slab mutant synaptic ultrastructural features

\begin{tabular}{|c|c|c|c|}
\hline Ultrastructural features & Wild type & slab & Significance \\
\hline Number of synaptic profiles scored & 39 & 24 & \\
\hline Mean terminal cross-sectional area $\left(\mu \mathrm{m}^{2}\right)$ & $1.50 \pm 0.14$ & $1.27 \pm 0.16$ & NS \\
\hline Mean SV density (total number of SVs per profile $/ \mu \mathrm{m}^{-2}$ ) & $46.0 \pm 3.6$ & $46.2 \pm 6.3$ & NS \\
\hline Number of clustered SVs ( $<250 \mathrm{~nm}$ from AZ) & $12.7 \pm 0.5$ & $12.5 \pm 0.6$ & NS \\
\hline Number of docked SVs at AZ & $2.1 \pm 0.2$ & $2.5 \pm 0.2$ & NS \\
\hline Number of nonclustered SVs ( $>250 \mu \mathrm{m}$ from AZ) & $43.0 \pm 3.9$ & $30.0 \pm 3.2$ & $p<0.05^{*}$ \\
\hline Number of linked SVs per profile & $3.5 \pm 0.6$ & $7.5 \pm 1.0$ & $p<0.002^{* *}$ \\
\hline Percentage of synaptic profiles with linked SVs & $57 \%(17 / 30)$ & $86 \%(19 / 22)$ & \\
\hline Percentage of linked SVs per profile & $6.0 \pm 1.0 \%$ & $17.7 \pm 2.4 \%$ & $p<0.0005^{* * *}$ \\
\hline Number SVs per section tethered to non-AZ membrane & $2.8 \pm 0.4(43)$ & $6.6 \pm 0.7(41)$ & $p<0.005^{* *}$ \\
\hline
\end{tabular}

Wild-type and slab null mutant $\left(s / a b^{7} / s_{l} a b^{3}\right)$ embryos were fixed and identically processed in parallel. Single sections exhibiting a clear AZ profile were quantified for each identified synapse. When multiple serial sections of the same synaps were obtained, the profile containing the most prominent AZ was selected for analysis. Analysis of tethered SVs included serially sectioned boutons (1-4 sections), with the mean number of tethered SVs per section scored for each bouton. The parentheses show the number of profiles examined were different from that in the first row. Values are mean \pm SEM. Asterisks indicate statistical significance determined by Mann-Whitney nonparametric test. NS, Not significant.

to threefold in slab mutant boutons compared with normal terminals ( $p<0.005$ vs wild type) (Table 1$)$. Linked and tethered SVs together represent $\sim 50 \%$ of the smaller nonclustered internal pool in slab bouton profiles, compared with $\sim 16 \%$ for wild type. These latter two phenotypes, which are both characterized by aberrantly increased SV sequestration, may reflect a related trafficking defect leading to an abnormal accumulation of a normal SV trafficking state.

\section{Discussion}

\section{Loss of Cdase impairs SV priming/fusion}

The slug-a-bed gene was identified in a forward screen for synaptic dysfunction mutants and encodes a long-chain Cdase that regulates the ceramide level. At slab mutant synapses, postsynaptic receptor function is unaltered, whereas nerve-evoked transmission and HO saline-evoked SV fusion is decreased by $50-$ $70 \%$. These results indicate a specific presynaptic impairment consistent with a loss of the RRP. However, slab synapses exhibit normal numbers of clustered and docked SVs at the AZ, ruling out defects in forming a localized releasable pool. We therefore conclude that the primary transmission impairment is a reduced ability of SVs to complete priming/fusion steps after docking.

Neurotransmitter release is most directly mediated by the RRP, a small cycling SV pool that includes a subpopulation of docked, fusion-competent vesicles at the AZ (Schikorski and Stevens, 1997, 2001; Sudhof, 2000; Mozhayeva et al., 2002; Richards et al., 2003; Rizzoli and Betz, 2004). Provided that the essential vesicle fusion machinery is functional, release efficacy is predictably correlated to the size of this docked pool (Dobrunz and Stevens, 1997; Reist et al., 1998; Pozzo-Miller et al., 1999; Delgado et al., 2000; Voets et al., 2001; Mozhayeva et al., 2002), which may be functionally assayed by hypertonic saline-evoked fusion (Stevens and Tsujimoto, 1995; Rosenmund and Stevens, 1996). This correlation is genetically supported by C. elegans mutations in the Sec-1-related protein UNC-18, which causes a parallel loss of docked SVs and hypertonic saline-evoked fusion (Weimer et al., 2003). Similarly, mutants in the mammalian UNC-18 homolog reduce docking and exocytosis of dense core secretory vesicles (Voets et al., 2001). Disruptions to downstream priming and fusion steps, including mutations in the SNARE (SNAP receptor) core complex proteins syntaxin-1, $N$-synaptobrevin, and SNAP-25 as well as in UNC-13 (uncoordinated-13) and RIM (Rab3-interacting molecule), which regulate SV priming, also severely reduce or eliminate $\mathrm{Ca}^{2+}$ - and hypertonic saline-evoked release (Broadie et al., 1995; Schulze et al., 1995; Aravamudan et al., 1999; Kidokoro, 2003). In contrast, however, these priming/ fusion mutants typically exhibit increased SV docking and a larger overall vesicle pool because of selective block of the exocytosis step(s) (Broadie et al., 1995; Aravamudan et al., 1999; Richmond and Broadie, 2002).

The slab mutant phenotypes place the functional transmission requirement downstream of docking at the level of vesicle priming/fusion, with the insignificant increase $(\sim 20 \%)$ in docked SVs consistent with a pronounced but incomplete inhibition of fusion. SNARE complex proteins have been shown to localize to sphingolipid- and sterol-enriched membrane raft environments in secretory cells (Chamberlain et al., 2001; Lang et al., 2001; Chamberlain and Gould, 2002). Other exocytic proteins, such as nSEC- 1 and $\alpha$ SNAP, are reported to localize to distinct sterolrich raft-like domains (Salaun et al., 2004). An accumulation and spatial misregulation of ceramide, the predominant sphingolipid in flies (Rietveld et al., 1999), is expected to disrupt the topology, lipid environment, and protein content of membrane exocytic domains. Ceramide has established roles regulating membrane domain fluidity and curvature, cholesterol aggregation, vesicle formation, and fusion (Li et al., 1999; Venkataraman and Futerman, 2000; van Blitterswijk et al., 2003). Increased membrane ceramide levels may therefore interfere with SV fusion competency by inhibiting lipid restructuring required for exocytosis, by inhibiting functional interactions between release machinery proteins, or by a combination of both lipid- and proteinmediated processes.

\section{Loss of Cdase increases SV pool sequestration}

Our results indicate that sphingolipids are also involved in regulating SV number and distribution. In slab mutant terminals, the endo/exo cycling pool labeled by FM1-43 is reduced by $\sim 30 \%$. Although overall mutant SV density is not changed, the number of SVs not localized to AZs is reduced by a similar percentage. Most interestingly, slab terminals exhibit a striking increase in the percentage of SV s linked together or tethered to the PM. Filamentous tethers often clearly appear to connect SVs and to connect SVs to the PM, singly and in multi-vesicle arrays. SVs sometimes appear both linked and tethered (Fig. $8 C, D$ ), suggesting that these forms of linkage are similar structurally and serve to sequester SVs from the AZ. Although previously overlooked by us and others (Prokop, 1999), normal synapses exhibit a small number of linked/tethered SVs, indicating they represent normal intermediate trafficking steps that are abnormally accumulated in the absence of SLAB.

C. elegans and Drosophila endophilin (endo) and synaptojanin (synj) mutants also exhibit prominent arrays or "strings" of linked SVs and SVs tethered to the PM by cytoskeletal filaments (Harris et al., 2000; Schuske et al., 2003; Verstreken et al., 2003). 
Both endo and synj SV trafficking phenotypes are associated with severe primary defects in endocytosis, resulting in a substantial depletion $(50-70 \%)$ of SVs from the entire terminal, including AZ regions, and an accumulation of intermediate endocytic structures at or near the PM (Harris et al., 2000; Guichet et al., 2002; Schuske et al., 2003; Verstreken et al., 2003). The qualitative similarities between the slab, endo, and synj phenotypes reinforce the conclusion that presynaptic lipid environments are important for regulating SV pool size, trafficking, and availability for fusion. However, the slab ultrastructural phenotype differs from these endocytic mutants in two significant respects. First, the reduction in vesicles is far less pronounced and restricted to non-AZ domains, whereas the clustered and docked populations appear unaffected. Second, the slab phenotype is more specific to an accumulation of later SV trafficking states.

How might SLAB Cdase and ceramide regulate SV trafficking? Ceramide resides both in plasma and vesicle membranes and is concentrated in raft domains, interacts with raft-associated proteins, and modulates general membrane endocytosis and trafficking (Brown and London, 2000; van Blitterswijk et al., 2003; Acharya et al., 2004) depending on its production and topological location in the membrane. Asymetrical generation of ceramide, as in rafts, promotes negative membrane curvature, vesicle budding, and vesicle aggregation (van Blitterswijk et al., 2003). Conversely, certain ceramide analogs inhibit membrane internalization and trafficking (Chen et al., 1995; Li et al., 1999). Ceramide may therefore have similar roles in synaptic PMs and SVs and is likely trafficked between the inner PM leaflet and the SV surface by endocytosis. SV recycling is thought to occur in specialized, spatially defined regions in which lipid and protein constituents are preassembled before vesicle budding (Martin, 2000). This process is potentially modulated by changes in PM ceramide levels and raft distribution. The slab ultrastructural and FM1-43 loading phenotypes do not indicate a severe endocytosis defect at the NMJ. However, given the pronounced SV fusion impairment in the absence of SLAB, a reduced level or spatial specificity of endocytosis may be sufficient to maintain a pool of SVs trafficked to release sites. A recent analysis of RRP organization at the frog NMJ suggests that, contrary to general assumption, the RRP may be widely dispersed throughout the overall vesicle population and not necessarily recruited from regions nearest the AZ (Rizzoli and Betz, 2004). If this is the case in Drosophila NMJ terminals, the increased SV sequestration observed in slab boutons could more directly underlie reduced RRP availability and weakened transmission, particularly during maintained activity. Raft domains have functions consistent with a role in SV tethering, including serving as sites for actin nucleation and polymerization and regulating actin cytoskeleton stability (Rozelle et al., 2000; Bruses et al., 2001). In the absence of SLAB, altered raft environments may inhibit F-actin-mediated vesicle trafficking or interactions between SV and the cytoskeletal scaffold. For example, disruption of membrane sphingolipid composition may interfere with SV binding to the tethering protein synapsin, which mediates the activity-dependent sequestration and mobilization of SVs (Chi et al., 2001, 2003).

\section{Cdase, ceramides, and lipid rafts in Drosophila}

Drosophila detergent-insoluble embryonic membranes are enriched in sphingolipids, sterols, and proteins (Rietveld et al., 1999), supporting the existence of functional raft domains in flies analogous to those in vertebrates. Drosophila sphingolipids consist predominantly of saturated long-chain ceramides, glycoceramides, and phosphoethanolamine ceramide (PECer), a sphin- gomyelin analog present in insects. Ceramide may therefore be produced in the membrane by PECer hydrolysis (Rietveld et al., 1999; Renault et al., 2002). Sterols constitute $\sim 18 \%$ of Drosophila membranes and over $30 \%$ of raft lipid fractions, relative to phospholipids (Rietveld et al., 1999). Cholesterol, an essential vertebrate membrane component, contributes only a fraction of sterols but may, nevertheless, have an important role in membrane and raft structure and in the regulation of proteins such as signaling protein Hedgehog, known to undergo cholesterol modification. Notably, Hedgehog and numerous glycosylphosphatidylinositol (GPI)-linked proteins are localized to Drosophila rafts (Rietveld et al., 1999), indicating that GPI linkage is a conserved mechanism for targeting proteins to rafts by (Tsui-Pierchala et al., 2002)

SLAB Cdase contains a secretory signal sequence and is secreted in an $\mathrm{N}$-glycosylated form when expressed in Drosophila S2 cells (Yoshimura et al., 2002). SLAB overexpression in the Drosophila eye suppresses retinal degeneration by reducing ceramide levels and facilitating membrane recycling (Acharya et al., 2003, 2004), consistent with our findings. We have used protein localization and mutant loss-of-function studies to examine expression and functional requirement of the endogenous Cdase for the first time. SLAB is expressed in numerous tissues and cells and prominently enriched in central neurons. Mutant lethality is rescued by neuronally targeted gene expression, showing that SLAB neuronal expression is essential. $\mathrm{C}_{5}$-ceramide, an analog known to be trafficked and metabolized in cells (Pagano et al., 2000 b), is rapidly accumulated at synaptic boutons, and its synaptic concentration is dependent on the slab expression level. These results suggest that endogenous sphingolipids are enriched and dynamically trafficked at Drosophila synapses and regulated by SLAB activity. SLAB may regulate synaptic sphinolipid environments by several plausible pathways. In neurons, SLAB is clearly cytoplasmically localized, consistent with either an intracellular or secreted function. SLAB may be present at low levels and function in the synaptic terminal; alternatively, it may be primarily secreted and act on the neuronal PM. If the essential protein function is secreted, however, our rescue results support the conclusion that neuronal rather than general secretion is required. Finally, SLAB may regulate sphingolipid production and content in membrane trafficked to and incorporated at the synapse.

Our results provide further evidence for the involvement of sphingolipid raft domains in specialized SV trafficking and exocytosis. A role for ceramide-rich membrane domains in SV priming or fusion processes represents the most direct potential involvement in neurotransmitter release. Likewise, sphinoglipiddependent interactions between SV and tethering proteins potentially regulate recycling and trafficking steps. Future studies will investigate mechanistic links between SLAB, lipid raft domains, and the established SV fusion and trafficking machinery.

\section{References}

Abrams JM, White K, Fessler LI, Steller H (1993) Programmed cell death during Drosophila embryogenesis. Development 117:29-43.

Acharya U, Patel S, Koundakjian E, Nagashima K, Han X, Acharya JK (2003) Modulating sphingolipid biosynthetic pathway rescues photoreceptor degeneration. Science 299:1740-1743.

Acharya U, Mowen MB, Nagashima K, Acharya JK (2004) Ceramidase expression facilitates membrane turnover and endocytosis of rhodopsin in photoreceptors. Proc Natl Acad Sci USA 101:1922-1926.

Adams MD, Celniker SE, Holt CA, Evans JD, Gocayne EA (2000) The genome sequence of Drosophila melanogaster. Science 287:2185-2195.

Aravamudan B, Fergestad T, Davis WS, Rodesch CK, Broadie K (1999) Dro- 
sophila Unc-13 is essential for synaptic transmission. Nat Neurosci 2:965-971.

Auld VJ, Fetter RD, Broadie K, Goodman CS (1995) Gliotactin, a novel transmembrane protein on peripheral glia, is required to form the bloodnerve barrier in Drosophila. Cell 81:757-767.

Benfenati F, Valtorta F, Chieregatti E, Greengard P (1992) Interaction of free and synaptic vesicle-bound synapsin I with F-actin. Neuron 8:377-386.

Beumer K, Matthies HJ, Bradshaw A, Broadie K (2002) Integrins regulate DLG/FAS2 via a CaM kinase II-dependent pathway to mediate synapse elaboration and stabilization during postembryonic development. Development 129:3381-3391.

Bloom O, Evergren E, Tomilin N, Kjaerulff O, Low P, Brodin L, Pieribone VA, Greengard P, Shupliakov O (2003) Colocalization of synapsin and actin during synaptic vesicle recycling. J Cell Biol 161:737-747.

Boquet I, Hitier R, Dumas M, Chaminade M, Preat T (2000) Central brain postembryonic development in Drosophila: implication of genes expressed at the interhemispheric junction. J Neurobiol 42:33-48.

Bose R, Chen P, Loconti A, Grullich C, Abrams JM, Kolesnick RN (1998) Ceramide generation by the Reaper protein is not blocked by the caspase inhibitor, p35. J Biol Chem 273:28852-28859.

Broadie K, Bate M (1993) Activity-dependent development of the neuromuscular synapse during Drosophila embryogenesis. Neuron 11:607-619.

Broadie K, Prokop A, Bellen HJ, O’Kane CJ, Schulze KL, Sweeney ST (1995) Syntaxin and synaptobrevin function downstream of vesicle docking in Drosophila. Neuron 15:663-673.

Broadie KS, Bate M (1991) The development of adult muscles in Drosophila: ablation of identified muscle precursor cells. Development 113:103-118.

Brown DA, London E (2000) Structure and function of sphingolipid- and cholesterol-rich membrane rafts. J Biol Chem 275:17221-17224.

Bruses JL, Chauvet N, Rutishauser U (2001) Membrane lipid rafts are necessary for the maintenance of the $(\alpha) 7$ nicotinic acetylcholine receptor in somatic spines of ciliary neurons. J Neurosci 21:504-512.

Ceccaldi PE, Grohovaz F, Benfenati F, Chieregatti E, Greengard P, Valtorta F (1995) Dephosphorylated synapsin I anchors synaptic vesicles to actin cytoskeleton: an analysis by videomicroscopy. J Cell Biol 128:905-912.

Chamberlain LH, Gould GW (2002) The vesicle- and target-SNARE proteins that mediate Glut4 vesicle fusion are localized in detergent-insoluble lipid rafts present on distinct intracellular membranes. J Biol Chem 277:49750-49754.

Chamberlain LH, Burgoyne RD, Gould GW (2001) SNARE proteins are highly enriched in lipid rafts in PC12 cells: implications for the spatial control of exocytosis. Proc Natl Acad Sci USA 98:5619-5624.

Chen CS, Rosenwald AG, Pagano RE (1995) Ceramide as a modulator of endocytosis. J Biol Chem 270:13291-13297.

Chi P, Greengard P, Ryan TA (2001) Synapsin dispersion and reclustering during synaptic activity. Nat Neurosci 4:1187-1193.

Chi P, Greengard P, Ryan TA (2003) Synaptic vesicle mobilization is regulated by distinct synapsin I phosphorylation pathways at different frequencies. Neuron 38:69-78.

Dbaibo GS, Hannun YA (1998) Signal transduction and the regulation of apoptosis: roles of ceramide. Apoptosis 3:317-334.

Delgado R, Maureira C, Oliva C, Kidokoro Y, Labarca P (2000) Size of vesicle pools, rates of mobilization, and recycling at neuromuscular synapses of a Drosophila mutant, shibire. Neuron 28:941-953.

Dobrunz LE, Stevens CF (1997) Heterogeneity of release probability, facilitation, and depletion at central synapses. Neuron 18:995-1008.

Eroglu C, Brugger B, Wieland F, Sinning I (2003) Glutamate-binding affinity of Drosophila metabotropic glutamate receptor is modulated by association with lipid rafts. Proc Natl Acad Sci USA 100:10219-10224.

Featherstone DE, Rushton EM, Hilderbrand-Chae M, Phillips AM, Jackson FR, Broadie K (2000) Presynaptic glutamic acid decarboxylase is required for induction of the postsynaptic receptor field at a glutamatergic synapse. Neuron 27:71-84.

Featherstone DE, Davis WS, Dubreuil RR, Broadie K (2001) Drosophila $\alpha$ and $\beta$-spectrin mutations disrupt presynaptic neurotransmitter release. J Neurosci 21:4215-4224.

Featherstone DE, Rushton E, Broadie K (2002) Developmental regulation of glutamate receptor field size by nonvesicular glutamate release. Nat Neurosci 5:141-146.

Fergestad T, Broadie K (2001) Interaction of stoned and synaptotagmin in synaptic vesicle endocytosis. J Neurosci 21:1218-1227.
Fergestad T, Wu MN, Schulze KL, Lloyd TE, Bellen HJ, Broadie K (2001) Targeted mutations in the syntaxin $\mathrm{H} 3$ domain specifically disrupt SNARE complex function in synaptic transmission. J Neurosci 21:9142-9150.

Greengard P, Valtorta F, Czernik AJ, Benfenati F (1993) Synaptic vesicle phosphoproteins and regulation of synaptic function. Science 259:780-785.

Greengard P, Benfenati F, Valtorta F (1994) Synapsin I, an actin-binding protein regulating synaptic vesicle traffic in the nerve terminal. Adv Second Messenger Phosphoprotein Res 29:31-45.

Grigliatti TA (1998) Transposons-gene tagging and mutagenesis. In: Drosophila: a practical approach, Ed 2 (Roberts DB, ed), pp 85-107. Oxford: IRL.

Guichet A, Wucherpfennig T, Dudu V, Etter S, Wilsch-Brauniger M, Hellwig A, Gonzalez-Gaitan M, Huttner WB, Schmidt AA (2002) Essential role of endophilin A in synaptic vesicle budding at the Drosophila neuromuscular junction. EMBO J 21:1661-1672.

Harris T, Hartweig E, Horvitz R, Jorgensen E (2000) Mutations in synaptojanin disrupt synaptic vesicle recycling. J Cell Biol 150:589-599.

Helms JB, Zurzolo C (2004) Lipids as targeting signals: lipid rafts and intracellular trafficking. Traffic 5:247-254.

Hering H, Lin CC, Sheng M (2003) Lipid rafts in the maintenance of synapses, dendritic spines, and surface AMPA receptor stability. J Neurosci 23:3262-3271.

Herr DR, Fyrst H, Phan V, Heinecke K, Georges R, Harris GL, Saba JD (2003) Sply regulation of sphingolipid signaling molecules is essential for Drosophila development. Development 130:2443-2453.

Hoekstra D, Maier O, van der Wouden JM, Slimane TA, van ISC (2003) Membrane dynamics and cell polarity: the role of sphingolipids. J Lipid Res 44:869-877.

Holley CL, Olson MR, Colon-Ramos DA, Kornbluth S (2002) Reaper eliminates IAP proteins through stimulated IAP degradation and generalized translational inhibition. Nat Cell Biol 4:439-444.

Kidokoro Y (2003) Roles of SNARE proteins and synaptotagmin I in synaptic transmission: studies at the Drosophila neuromuscular synapse. Neurosignals 12:13-30.

Kuromi H, Kidokoro Y (1998) Two distinct pools of synaptic vesicles in single presynaptic boutons in a temperature-sensitive Drosophila mutant shibire. Neuron 20:917-925.

Kuromi H, Kidokoro Y (1999) The optically determined size of exo/endo cycling vesicle pool correlates with the quantal content at the neuromuscular junction of Drosophila larvae. J Neurosci 19:1557-1565.

Kuromi H, Kidokoro Y (2000) Tetanic stimulation recruits vesicles from reserve pool via a cAMP-mediated process in Drosophila synapses. Neuron 27:133-143.

Lafont F, Verkade P, Galli T, Wimmer C, Louvard D, Simons K (1999) Raft association of SNAP receptors acting in apical trafficking in Madin-Darby canine kidney cells. Proc Natl Acad Sci USA 96:3734-3738.

Lai EC (2003) Lipid rafts make for slippery platforms. J Cell Biol 162:365-370.

Lang T, Bruns D, Wenzel D, Riedel D, Holroyd P, Thiele C, Jahn R (2001) SNAREs are concentrated in cholesterol-dependent clusters that define docking and fusion sites for exocytosis. EMBO J 20:2202-2213.

Li R, Blanchette-Mackie EJ, Ladisch S (1999) Induction of endocytic vesicles by exogenous C(6)-ceramide. J Biol Chem 274:21121-21127.

Lipsky NG, Pagano RE (1985) Intracellular translocation of fluorescent sphingolipids in cultured fibroblasts: endogenously synthesized sphingomyelin and glucocerebroside analogues pass through the Golgi apparatus en route to the plasma membrane. J Cell Biol 100:27-34.

Luo L, Liao YJ, Jan LY, Jan YN (1994) Distinct morphogenetic functions of similar small GTPases: Drosophila Dracl is involved in axonal outgrowth and myoblast fusion. Genes Dev 8:1787-1802.

Maekawa S, Iino S, Miyata S (2003) Molecular characterization of the detergent-insoluble cholesterol-rich membrane microdomain (raft) of the central nervous system. Biochim Biophys Acta 1610:261-270.

Martin TF (2000) Racing lipid rafts for synaptic-vesicle formation. Nat Cell Biol 2:E9-E11.

McMaster CR (2001) Lipid metabolism and vesicle trafficking: more than just greasing the transport machinery. Biochem Cell Biol 79:681-692.

Merrill Jr AH, Schmelz EM, Dillehay DL, Spiegel S, Shayman JA, Schroeder JJ, Riley RT, Voss KA, Wang E (1997) Sphingolipids-the enigmatic lipid class: biochemistry, physiology, and pathophysiology. Toxicol Appl Pharmacol 142:208-225.

Mitter D, Reisinger C, Hinz B, Hollmann S, Yelamanchili SV, Treiber-Held S, Ohm TG, Herrmann A, Ahnert-Hilger G (2003) The synaptophysin/ 
synaptobrevin interaction critically depends on the cholesterol content. J Neurochem 84:35-42.

Mozhayeva MG, Sara Y, Liu X, Kavalali ET (2002) Development of vesicle pools during maturation of hippocampal synapses. J Neurosci 22:654-665.

O'Kane C (1998) Enhancer traps. In: Drosophila: a practical approach, Ed 2 (Roberts DB, ed), pp 131-178. Oxford: IRL.

Pagano RE, Chen CS (1998) Use of BODIPY-labeled sphingolipids to study membrane traffic along the endocytic pathway. Ann NY Acad Sci 845:152-160.

Pagano RE, Sleight RG (1985) Defining lipid transport pathways in animal cells. Science 229:1051-1057.

Pagano RE, Sepanski MA, Martin OC (1989) Molecular trapping of a fluorescent ceramide analogue at the Golgi apparatus of fixed cells: interaction with endogenous lipids provides a trans-Golgi marker for both light and electron microscopy. J Cell Biol 109:2067-2079.

Pagano RE, Martin OC, Kang HC, Haugland RP (1991) A novel fluorescent ceramide analogue for studying membrane traffic in animal cells: accumulation at the Golgi apparatus results in altered spectral properties of the sphingolipid precursor. J Cell Biol 113:1267-1279.

Pagano RE, Puri V, Dominguez M, Marks DL (2000a) Membrane traffic in sphingolipid storage diseases. Traffic 1:807-815.

Pagano RE, Watanabe R, Wheatley C, Dominguez M (2000b) Applications of BODIPY-sphingolipid analogs to study lipid traffic and metabolism in cells. Methods Enzymol 312:523-534.

Paratcha G, Ibanez CF (2002) Lipid rafts and the control of neurotrophic factor signaling in the nervous system: variations on a theme. Curr Opin Neurobiol 12:542-549.

Petersen SA, Fetter RD, Noordermeer JN, Goodman CS, DiAntonio A (1997) Genetic analysis of glutamate receptors in Drosophila reveals a retrograde signal regulating presynaptic transmitter release. Neuron 19:1237-1248.

Pozzo-Miller LD, Gottschalk W, Zhang L, McDermott K, Du J, Gopalakrishnan R, Oho C, Sheng ZH, Lu B (1999) Impairments in high-frequency transmission, synaptic vesicle docking, and synaptic protein distribution in the hippocampus of BDNF knockout mice. J Neurosci 19:4972-4983.

Prokop A (1999) Integrating bits and pieces: synapse structure and formation in Drosophila embryos. Cell Tissue Res 297:169-186.

Pronk GJ, Ramer K, Amiri P, Williams LT (1996) Requirement of an ICElike protease for induction of apoptosis and ceramide generation by REAPER. Science 271:808-810.

Putz U, Schwarzmann G (1995) Golgi staining by two fluorescent ceramide analogues in cultured fibroblasts requires metabolism. Eur J Cell Biol 68:113-121.

Reist NE, Buchanan J, Li J, DiAntonio A, Buxton EM, Schwarz TL (1998) Morphologically docked synaptic vesicles are reduced in synaptotagmin mutants of Drosophila. J Neurosci 18:7662-7673.

Renault AD, Starz-Gaiano M, Lehmann R (2002) Metabolism of sphingosine 1-phosphate and lysophosphatidic acid: a genome wide analysis of gene expression in Drosophila. Mech Dev 119 [Suppl 1]:S293-S301.

Richards DA, Guatimosim C, Rizzoli SO, Betz WJ (2003) Synaptic vesicle pools at the frog neuromuscular junction. Neuron 39:529-541.

Richmond JE, Broadie KS (2002) The synaptic vesicle cycle: exocytosis and endocytosis in Drosophila and C. elegans. Curr Opin Neurobiol 12:499-507.

Rietveld A, Neutz S, Simons K, Eaton S (1999) Association of sterol- and glycosylphosphatidylinositol-linked proteins with Drosophila raft lipid microdomains. J Biol Chem 274:12049-12054.

Riezman H, van Meer G (2004) Lipid pickup and delivery. Nat Cell Biol 6:15-16.

Rizzoli SO, Betz WJ (2004) The structural organization of the readily releasable pool of synaptic vesicles. Science 303:2037-2039.

Rosenmund C, Stevens CF (1996) Definition of the readily releasable pool of vesicles at hippocampal synapses. Neuron 16:1197-1207.

Rozelle AL, Machesky LM, Yamamoto M, Driessens MH, Insall RH, Roth MG, Luby-Phelps K, Marriott G, Hall A, Yin HL (2000) Phosphatidylinositol 4,5-bisphosphate induces actin-based movement of raft-enriched vesicles through WASP-Arp2/3. Curr Biol 10:311-320.

Rubin GM, Hong L, Brokstein P, Evans-Holm M, Frise E, Stapleton M, Harvey DA (2000) A Drosophila complementary DNA resource. Science 287:2222-2224.
Saitoe M, Tanaka S, Takata K, Kidokoro Y (1997) Neural activity affects distribution of glutamate receptors during neuromuscular junction formation in Drosophila embryos. Dev Biol 184:48-60.

Salaun C, James DJ, Chamberlain LH (2004) Lipid rafts and the regulation of exocytosis. Traffic 5:255-264.

Sankaranarayanan S, Atluri PP, Ryan TA (2003) Actin has a molecular scaffolding, not propulsive, role in presynaptic function. Nat Neurosci 6:127-135.

Schikorski T, Stevens CF (1997) Quantitative ultrastructural analysis of hippocampal excitatory synapses. J Neurosci 17:5858-5867.

Schikorski T, Stevens CF (2001) Morphological correlates of functionally defined synaptic vesicle populations. Nat Neurosci 4:391-395.

Schulze KL, Broadie K, Perin MS, Bellen HJ (1995) Genetic and electrophysiological studies of Drosophila syntaxin-1A demonstrate its role in nonneuronal secretion and neurotransmission. Cell 80:311-320.

Schuske KR, Richmond JE, Matthies DS, Davis WS, Runz S, Rube DA, van der Bliek AM, Jorgensen EM (2003) Endophilin is required for synaptic vesicle endocytosis by localizing synaptojanin. Neuron 40:749-762.

Spiegel S, Cuvillier O, Edsall LC, Kohama T, Menzeleev R, Olah Z, Olivera A, Pirianov G, Thomas DM, Tu Z, Van Brocklyn JR, Wang F (1998) Sphingosine-1-phosphate in cell growth and cell death. Ann NY Acad Sci 845:11-18

Stevens CF, Tsujimoto T (1995) Estimates for the pool size of releasable quanta at a single central synapse and for the time required to refill the pool. Proc Natl Acad Sci USA 92:846-849.

Sudhof TC (2000) The synaptic vesicle cycle revisited. Neuron 28:317-320.

Suzuki T, Ito J, Takagi H, Saitoh F, Nawa H, Shimizu H (2001) Biochemical evidence for localization of AMPA-type glutamate receptor subunits in the dendritic raft. Brain Res Mol Brain Res 89:20-28.

Tani M, Iida H, Ito M (2003) O-glycosylation of mucin-like domain retains the neutral ceramidase on the plasma membranes as a type II integral membrane protein. J Biol Chem 278:10523-10530.

Taverna E, Saba E, Rowe J, Francolini M, Clementi F, Rosa P (2004) Role of lipid microdomains in P/Q-type calcium channel (Cav2.1) clustering and function in presynaptic membranes. J Biol Chem 279:5127-5134.

The Flybase Consortium (2003) The Flybase database of the Drosophila genome projects and community literature. Nucleic Acids Res 31:172-175

Thiele C, Hannah MJ, Fahrenholz F, Huttner WB (2000) Cholesterol binds to synaptophysin and is required for biogenesis of synaptic vesicles. Nat Cell Biol 2:42-49.

Tsui-Pierchala BA, Encinas M, Milbrandt J, Johnson Jr EM (2002) Lipid rafts in neuronal signaling and function. Trends Neurosci 25:412-417.

van Blitterswijk WJ, van der Luit AH, Veldman RJ, Verheij M, Borst J (2003) Ceramide: second messenger or modulator of membrane structure and dynamics? Biochem J 369:199-211.

van Meer G, Holthuis JC (2000) Sphingolipid transport in eukaryotic cells Biochim Biophys Acta 1486:145-170.

Venkataraman K, Futerman AH (2000) Ceramide as a second messenger: sticky solutions to sticky problems. Trends Cell Biol 10:408-412.

Verstreken P, Koh TW, Schulze KL, Zhai RG, Hiesinger PR, Zhou Y, Mehta SQ, Cao Y, Roos J, Bellen HJ (2003) Synaptojanin is recruited by endophilin to promote synaptic vesicle uncoating. Neuron 40:733-748.

Voets T, Toonen RF, Brian EC, de Wit H, Moser T, Rettig J, Sudhof TC, Neher E, Verhage M (2001) Munc18-1 promotes large dense-core vesicle docking. Neuron 31:581-591.

Watanabe R, Funato K, Venkataraman K, Futerman AH, Riezman H (2002) Sphingolipids are required for the stable membrane association of glycosylphosphatidylinositol-anchored proteins in yeast. J Biol Chem 277:49538-49544

Weimer RM, Richmond JE, Davis WS, Hadwiger G, Nonet ML, Jorgensen EM (2003) Defects in synaptic vesicle docking in unc-18 mutants. Nat Neurosci 6:1023-1030.

Wodarz A, Hinz U, Engelbert M, Knust E (1995) Expression of crumbs confers apical character on plasma membrane domains of ectodermal epithelia of Drosophila. Cell 82:67-76.

Yoshihara M, Suzuki K, Kidokoro Y (2000) Two independent pathways mediated by cAMP and protein kinase A enhance spontaneous transmitter release at Drosophila neuromuscular junctions. J Neurosci 20:8315-8322.

Yoshimura Y, Okino N, Tani M, Ito M (2002) Molecular cloning and characterization of a secretory neutral ceramidase of Drosophila melanogaster. J Biochem (Tokyo) 132:229-236. 